Malou Haine

\title{
Une nouvelle source d'archives pour identifier les marques de fabrique de facteurs d'instruments de musique (1860 à 1919)
}

Introduction ${ }^{\mathrm{I}} \quad$ En mai 20I4 s'ouvrait la base de données intitulée Marques d'instruments de musique (I860-I9I9) à l'adresse web suivante: http://iremus.huma-num.fr/marquesinstruments-musique. ${ }^{2}$ Il s'agit des marques de fabriques des facteurs d'instruments de musique déposées au greffe du Tribunal de Commerce de Paris de I860 à I9I9 et conservées aux Archives de Paris. J'avais collecté ces marques lors de la préparation de mon doctorat à la fin des années I970 et ne les avais jamais exploitées depuis lors. Cette base de données, dont je suis la responsable scientifique, a pu être élaborée grâce à l'équipe de réalisation de l'IRPMF, composée d'Alban Framboisier, ingénieur d'étude CNRS et de Philippe Pigeon, administrateur systèmes et réseaux, placés sous la direction de Florence Gétreau, alors directrice de l'IRPMF. ${ }^{3}$

Je présenterai d'abord la loi française de I8 57 qui régit l'enregistrement officiel (mais facultatif) des marques de fabrique dans leur ensemble, pour ensuite me pencher plus précisément sur celles relatives aux instruments de musique, toutes catégories confondues. J'expliquerai les limites temporelles de mon étude. Les facteurs, luthiers et marchands concernés sont principalement installés à Paris, mais plusieurs fabricants sont domiciliés en province; des étrangers déposent aussi leurs marques à Paris. Je m'attacherai ensuite au contenu et aux caractéristiques propres aux marques d'instruments de musique. Pour conclure, je donnerai quelques éléments relatifs au fonctionnement de la base de données.

La loi française du $\mathbf{2 3}$ juin $\mathbf{1 8 5 7}$ Bien avant le XIX siècle, les facteurs d'instruments de musique ont appliqué une marque distinctive sur les instruments qu'ils fabriquaient. Après la disparition des corporations à la fin de l'Ancien Régime, la législation sur les marques de fabrique reste assez floue et incomplète jusqu'au milieu du XIX ${ }^{\text {e }}$ siècle. ${ }^{4}$ Certes, le nom commercial est fixé par la loi du 28 juillet I824, les dessins et modèles de

1 Les appels de note placés après la ponctuation sont dus à l'éditeur allemand et non à l'auteur de cet article.

2 Pour citer cette base de données : Malou Haine : Marques d'instruments de musique (I860-I9I9), http:// iremus.huma-num.fr/marques-instruments-musique/ - suivi de la date de consultation.

3 Depuis la rédaction de cet article, l'Irpmf a été intégré dans l'IreMus - Institut de recherche en musicologie (Paris).

4 Alain Beltran/Sophie Chauveau/Gabriel Galvez-Behar: Des brevets et des marques. Une histoire de la propriété industrielle, Paris 200I, p. 90. 
fabrique par l'ordonnance des 27-29 août I825, mais c'est la loi du 23 juin I857 sur les marques de fabrique ou de commerce qui fixe pour longtemps la législation. Celle-ci est donc la même pour la marque industrielle et la marque commerciale. ${ }^{5}$

Selon la loi, la marque de fabrique est facultative, ${ }^{6}$ moyennant le paiement d'un droit fixe de I franc auquel s'ajoutent les frais de timbre et d'enregistrement auprès du greffe du tribunal de son domicile. En d'autres termes, l'enregistrement s'effectue à l'échelon local. Trois exemplaires du modèle doivent être déposés, ainsi qu'un cliché typographique. Celui-ci est rendu au déposant après sa publication par l'Office National de la Propriété Industrielle.7 Tout comme pour le dépôt de brevet, il n'y a pas d'examen préalable lors du dépôt d'une marque qui reste valable pour une période de quinze ans, renouvelable à l'envi par d'autres dépôts, contrairement à la durée des brevets qui est limitée dans le temps. En cas de contrefaçon, les peines sont très lourdes, allant jusqu'à 3000 francs et à trois ans d'emprisonnement, selon la gravité des fautes commises, domaine qui ne nous intéresse pas dans le cadre de cet article.

La législation reconnait diverses formes à la marque de fabrique : «Les noms sous une forme distinctive, les dénominations, emblèmes, empreintes, timbres, cachets, vignettes, reliefs, lettres, chiffres, enveloppes et tous les autres signes servant à distinguer les produits d'une fabrique ou les objets d'un commerce » (art. I). Nous constaterons plus avant les différentes formes que peuvent prendre les marques déposées par les facteurs et marchands d'instruments de musique.

Les marques de fabrique relatives à la musique Ma recherche porte sur les marques de fabrique déposées au greffe du Tribunal de Commerce de la Seine après la promulgation de la loi de 1857 et conservées aux Archives de Paris. ${ }^{8}$ Les premiers enregistrements de marques en général datent de I858, mais celles concernant la musique ne commencent qu'en mai I860. J'ai étendu ma recherche jusqu'en I9I9, couvrant ainsi soixante années

5 Si les facteurs d'instruments de musique sont à la fois fabricants et commerçants, ces deux professions se scindent peu à peu vers 1860 ; voir Malou Haine : Les facteurs d'instruments de musique à Paris au XIXe siècle. Des artisans face à l'industrialisation, Bruxelles I985.

6 Des règlements d'administration publique peuvent néanmoins déclarer obligatoire le dépôt de marque pour certains produits (article I de la loi du 23 juin I857 sur les marques de fabrique ou de commerce).

7 L'Office National de la Propriété Industrielle s'est transformé en INPI (Institut de la Propriété Industrielle) en I951.

8 Les Archives de Paris conservent les archives du Tribunal de Commerce de la Seine - Tribunal de Commerce de Paris, Propriété industrielle : marques de fabrique, séries DI8U3. Ces documents ont été consultés et photographiés au début des années I980, à l'époque où ces Archives se trouvaient encore au Quai Henri IV et s'intitulaient «Archives de la Seine ». Les volumes consultés étaient alors dépourvus de cotes. 
de dépôts, date limite qui correspond également à l'examen de volumes complets déposés aux Archives de Paris.

La classe 43 comprend les marques relatives aux instruments de musique et aux instruments de précision: dans un même volume d'archives se côtoient donc des marques de déposants qui s'occupent d'instruments de mathématique, de physique, de chimie, d'optique et de lunetterie, de poids et mesures. Certains industriels dont l'activité principale n'a rien à voir avec la facture instrumentale s'y intéressent néanmoins (voir plus avant).

Précisons tout d'abord la présentation matérielle des dépôts de marque. Ces marques sont conservées dans de grands et gros volumes de format bible, comprenant deux ou trois marques par page, collées au fur et à mesure de leur enregistrement dans un ordre plus ou moins chronologique. Insérée dans un rectangle, chaque marque se trouve placée au centre d'une fiche : voir les deux exemples de fiches à la page suivante. De part et d'autre de la marque se trouvent généralement (mais ce n'est pas toujours le cas) des informations nominatives et descriptives qui la concernent.

À gauche, figure la légende de la marque ou sa description sommaire. À droite sont indiquées les données administratives : ville du greffe, numéro d'ordre du greffe, date et heure du dépôt, nom du déposant ou de son mandataire ; sont ensuite décrites la destination de la marque (par exemple «modèle d'un poinçon destiné à être appliqué sur les cymbales ») et la manière de l'appliquer (par exemple : «à imprimer en creux »).

Les signatures du déposant ou de son mandataire, ainsi que celle du greffier, figurent à la fois à gauche et à droite au bas du document. Jusqu'en décembre I890, chaque marque porte un numéro d'enregistrement (selon la succession des marques déposées au greffe) appliqué à l'aide d'un tampon encreur numéroteur. Sans doute ce numéro a-t-il été considéré ensuite comme superflu puisque le numéro d'ordre du greffe est suffisant pour l'identifier, car le numéro encreur disparaît à partir de février i89i.

Les dépôts restent longtemps rédigés à la main. Bien que la machine à écrire se soit propagée en France vers I885, le premier document dactylographié d'un dépôt de marque concernant la facture instrumentale date de I898. Si la pratique d'utiliser des documents dactylographiés se répand, elle ne s'intensifie qu'aux environs de i902, mais elle ne sera pas encore généralisée en I9I9. En revanche, les sociétés de mandataires utilisent de plus en plus des documents pré-imprimés pour les données récurrentes.

Qui s'occupe de faire la démarche administrative auprès du greffe du tribunal de commerce ? Dans les petites entreprises, c'est souvent le patron, son fils ou un employé, tandis que les étrangers et les facteurs de province font appel à des mandataires, soit une personne de leur choix, soit, et c'est le cas le plus fréquent, à des sociétés spécialisées en matière de propriété industrielle. Ces mandataires déposent des marques pour différents fabricants, et leurs noms se retrouvent au fil des années pour d'autres déposants. Ils 
ILLUSTRATION I Fiche manuscrite $n^{\circ} 259$ : dépôt de la marque «A NICOLAS LUPOT » par Gustave Bernardel, comprenant la photographie de Nicolas Lupot, 13 décembre 1900. Marque destinée « à désigner tous articles de lutherie et instruments de musique »

Illustration 2 Fiche imprimée $n^{\circ} 1045$ : dépôt de la marque « VOLUNOME » par Émile Bert, 19 janvier 1915. Marque destinée « à des instruments de musique [...] et autres appareils reproducteurs de sons »
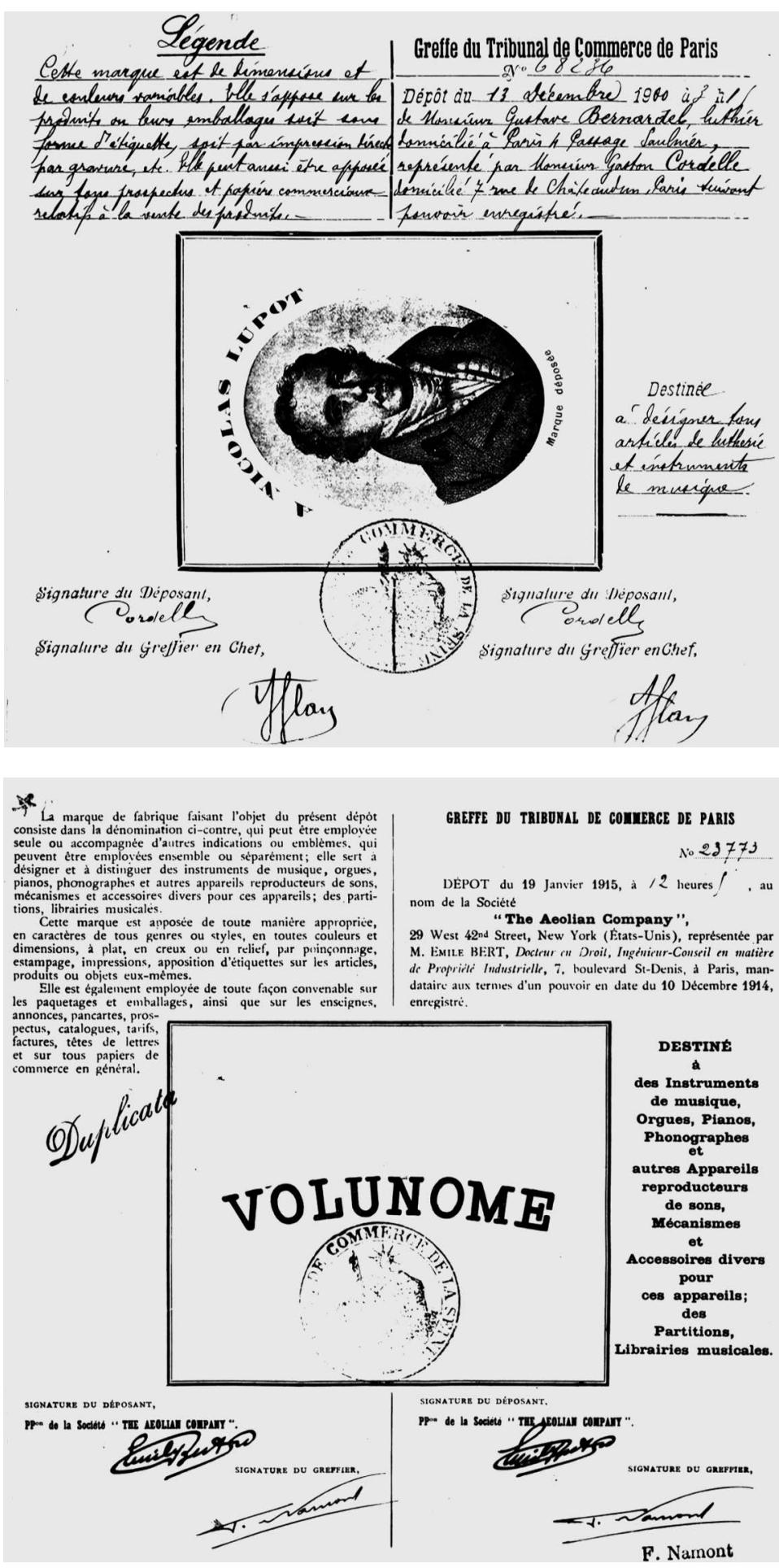
effectuent très souvent plusieurs dépôts un même jour. Une seule femme est recensée dans la base de données : Cécile Besson s'affirme en précisant qu'elle est « BREVETÉE », marquant bien ainsi l'accord au féminin de cet adjectif qui se conjugue principalement au masculin (fiche 98).

Le domaine retenu dans l'inventaire chronologique 9 des marques couvre la musique au sens large, comprenant principalement des marques destinées à des instruments de musique, des pièces détachées (touches de piano, pavillons, embouchures, etc.) ou à des accessoires (colophane, cordes, etc). Mais j'y ai également inclus des marques destinées à des méthodes didactiques, du papier ou boîte d'emballage, du papier à en-tête, des prospectus, annonces, etc.

À la fin des années I890, on voit apparaître, puis se multiplier, les marques destinées aux machines parlantes à cylindres ou à disques. Nul n'ignore que l'année i877 marque le point de départ des machines reproductrices de son grâce aux inventions du Français Charles Cros (le paléophone) et de l'Américain Thomas Edison (le phonographe), chacun travaillant de son côté sans concertation. Selon les marques déposées à Paris, les premières appellations de ces appareils apparaissent vingt ans plus tard : « phonographe et graphophone » (I6 mars I898, fiche 2II), «phonogramme» (6 avril I898, fiche 2I4), «machine parlante» (I6 février I899, fiche 225), «gramophone» (20 novembre I903, fiche 38I). ${ }^{\text {IO }}$ Les premières marques rivalisent de noms particuliers : «Echo », « Eden », «Eureka », «Le Mégaphone», « Motophone », « Musola», « Le Stentor», etc.

Le début du $\mathrm{Xx}^{\mathrm{e}}$ siècle assiste aussi à la prolifération de marques destinées aux pianos et harmoniums mécaniques : les premières marques sont déposées en I90I par la compagnie Aeolian de New York (The Aeolian Company). Ici aussi, les marques se multiplient : «Idéal orchestre», «Mignon», «Pianella», «Pianina», «Piano-Musica», «Opheola», «Orchestrelle», «Pédala-Piano», «Pleyela», «Polyphon», «Phonola», etc. avant que celui de «Pianola» ne finisse par s'identifier à tout piano mécanique et devienne un nom commun.

Les premiers instruments électriques sont également répertoriés, avec notamment la marque «Electrelle». Il faut néanmoins se méfier des descriptions dans lesquelles figure le terme «électrique», car celui-ci s'applique à l'action utilisée pour mettre en mouvement les pianos mécaniques, et non pour une quelconque génération électronique des sons. Ces nouveaux instruments n'existent pas encore à l'époque qui nous intéresse ici.

9 Le moteur de recherche propre à la base de données permet des recherches diverses.

10 Ne sont pas reprises dans nos inventaires les marques des machines destinées à reproduire le son au cinéma, et pour n'en citer que quelques-unes, par exemple celles du graphophonoscope (I896), de l'audiomètre Dussaud (I897) ou du chronophone (I902). 
Contrairement à d'autres fabricants, les facteurs d'instruments de musique ne se précipitent pas pour faire enregistrer leurs marques après la promulgation de la loi de I857. Leurs premiers dépôts datent de l'année I860 et sont assez rares durant cette première décennie, avec une ou deux marques par an ou à peine davantage. Au cours des décennies I870 et I880, les dépôts de marque restent peu nombreux, mais s'intensifient légèrement dans les années I89o; ils se multiplient ensuite considérablement dans les deux premières décennies du xxe siècle, comme le montrent les données statistiques ci-après :

$\begin{array}{cc}\text { années I860-I869 } & \text { I8 marques déposées } \\ \text { année I860 } & 2 \text { marques } \\ \text { année I86I } & \text { pas de marque } \\ \text { année I862 } & 2 \text { marques } \\ \text { année I863 } & \text { I marque } \\ \text { année I864 } & \text { I marque } \\ \text { année I865 } & 2 \text { marques } \\ \text { année I866 } & 6 \text { marques } \\ \text { année I867 } & \text { I marque } \\ \text { année I868 } & \text { I marque } \\ \text { année I869 } & 2 \text { marques } \\ \text { années I870-I879 } & 42 \text { marques } \\ \text { années I880-I889 } & 67 \text { marques } \\ \text { années I890-I899 } & \text { I09 marques } \\ \text { années I900-I9I0 } & 359 \text { marques } \\ \text { années I9II-I9I9 } & 577 \text { marques }\end{array}$

Il va de soi que les premières marques déposées entérinent leur usage antérieur. Il ne faudrait donc pas considérer ces premières dates de dépôt comme la date de leur mise en circulation. Par la suite (mais à quel moment exactement ?), les dépôts de marque correspondront à leurs premières utilisations. Il semble évident que lors d'un rachat ou d'une fusion, les nouveaux propriétaires ou directeurs s'empressent de faire enregistrer les marques existantes. Citons, entre autres, Joseph Honoré Derazey et son fils Just, installés à Mirecourt, qui lorsqu'ils rachètent en r864 le fonds de Didier Nicolas, ${ }^{\text {II }}$ déposent la marque de ce dernier, «A la ville de Crémone / D :Nicolas aîné/ A. Mirecourt », afin de l'utiliser à leur tour. De même, les alliances diverses du luthier Auguste Laberte et de ses descendants se traduisent par des raisons sociales différentes, donnant lieu à des marques tout aussi variées : Auguste Laberte deviendra Laberte-Humbert, Laberte-Humbert Frères, Laberte-Humbert Frères Fourier Magnié. 
Si les petits artisans se contentent d'une seule marque pour distinguer leurs produits, on constate néanmoins qu'un même facteur (ou une société), pour peu qu'il intensifie ou diversifie sa production, enregistre souvent plusieurs marques différentes, non seulement au fil des années, mais aussi en une seule démarche à l'occasion de dépôts groupés. Ces diverses marques servent, en principe, à distinguer les différents types de produits d'une même maison. Or, les descriptions fournies lors des dépôts ne précisent que très rarement les différences de destination. Augustin Bosio utilise deux marques différentes pour ses clarinettes, selon qu'il les applique sur des anches ou sur des becs : d'une part, la marque « CHANTECLER / LE COQ / A. Bosio » (fiche 6OI); d'autre part, « BEC CHANTECLAIR / A. BOSIO / MARSEILLE » (fiche 602). On remarquera l'orthographe différente : Chanteclair dans un cas, Chantecler dans l'autre. De son côté, Gautrot-Marquet distingue la qualité de ses instruments par des marques différentes selon qu'il s'agisse d'instruments de premier ou second choix (fiches 28 à 3I).

Malheureusement, la plupart des facteurs sont rarement aussi explicites que Bosio ou Gautrot-Marquet: le 25 mars I9I0, la maison Couesnon et $C^{\text {ie }}$ dépose six marques différentes destinées à des « séries SC », « séries HN », etc., sans qu'il soit possible, par la seule lecture de la fiche de dépôt, de déterminer les spécificités de ces diverses séries (fiches 6I4 à 6I9). ${ }^{\mathrm{I2}}$ En octobre I875, la Société des orgues Alexandre père et fils dépose quatre marques différentes, toutes destinées aux « orgues, pianos, etc. » : «Alexandre», «Alexandre Père », «Alexandre Fils », «Alexandre Père et Fils » (fiches 35 à 38). En I89o, la société renouvelle ces quatre marques en y ajoutant la ville « Paris » sur chacune d'elles (fiches I39 à I42). On constate ici que ces marques s'utilisent simultanément et non successivement dans le temps au gré des successions. Il en va de même des marques «STEINWAY \& SONS » et « STEINWAY», enregistrées toutes deux le I5 mai I886 (fiches 85 et 86).

Et l'on reste perplexe lorsque plusieurs marques différentes d'une même maison sont toutes destinées « aux instruments de musique », sans aucune autre précision. Prenons au hasard les deux marques déposées par Camille Thibouville le 2 novembre I909 (fiches 589 et 590) ou la dizaine de marques du luthier Charles Bailly, déposées le 5 août I9II et qui sont à appliquer «sur tous les violons sortant de son établissement». Ne s'agit-il pas dans certains cas de faire illusion aux clients potentiels en prétendant représenter plusieurs maisons ? Quelques sociétés ne se préoccupent même pas de préciser pour quels produits ils déposent une marque : ils mentionnent simplement que leur marque est destinée à «tout type de produits» de la classe 43 (voir notamment les fiches 593, 594, 845, 866, etc.). 
Ce sont principalement des luthiers, facteurs d'instruments ou marchands qui sont les dépositaires de ces marques : dans une moindre mesure, des fabricants de pièces détachées, ${ }^{\mathrm{I3}}$ des éditeurs de musique ${ }^{\mathrm{I} 4}$ des professeurs ${ }^{\mathrm{I} 5}$ ou de simples individus peuvent également être concernés. Enfin, plusieurs entreprises s'occupent accessoirement d'instruments de musique, alors que leurs raisons sociales explicitent très clairement leurs domaines d'exploitation. Donnons ici plusieurs exemples qui témoignent de la prospérité des instruments de musique au XIXe siècle et qui incitent de nombreuses sociétés à se diversifier et à ouvrir un nouveau secteur d'activités : les Aciéries \& Forges de Firminy ${ }^{16}$ (fiches 75 et 578), tout comme les Forges de Châtillon \& Commentry (fiche 83), fabriquent des fils métalliques pour cordes de pianos ; les Ateliers de Construction RF (Régy Frères) sont spécialisés dans la construction d'hélices pour avions, mais déposent des marques pour pièces détachées d'instruments de musique, et plus spécialement pour des machines destinées à fabriquer des pianos (fiches II23, II24, II67); la Société des armes et cycles de Saint-Étienne fabrique des violons sous la marque « San Stefano » (fiche roII) et des mandolines sous celle de "Sorrenlina» (fiche ror2). La marque nominative de l'Usine hydraulique des Forces motrices du Madon est exploitée par Jérôme Thibouville-Lamy pour « tous instruments et objets de musique, cordes harmoniques, phonographes, etc. » (fiche 359); la Société générale de Coutellerie \& Orfevrerie utilise une seule et même marque identique, «Apollo", tout à la fois pour des instruments de musique, des cartons perforés, des appareils de physique et de chimie, des machines à écrire, etc. (fiche I057); enfin, la Société des Boissons hygiéniques dépose la marque « KUB » pour des instruments de musique, accessoires et appareils de physique et de chimie, etc. (fiche 603).

Aujourd'hui encore, l'industrie reste intéressée par la facture instrumentale : la société automobile Peugeot Design Lab a conçu un piano avant-gardiste Pleyel. Ce piano demi-queue de nouvelle génération, utilisant de la fibre carbone pour le monopied central et le couvercle, a été exposé au salon de ... l'automobile !'7

13 Citons par exemple Gérard et fils pour des touches de pianos (fiche 50), Charles Monti pour claviers de pianos (fiche 44).

Citons notamment Alphonse Michel avec sa marque « La Solidarité musicale » (fiche 95); Henri Heugel avec celle de «Au Ménestrel» (fiche ro3).

15 Henri Tauay, professeur de musique, dépose une marque pour des cordes à son nom; il se dit également « fabricant de violons » (fiche 46). Victor Charles Paul Durup de $S^{t}$ Paul dépose une marque pour un cadran harmonique permettant de moduler instantanément dans tous les tons (fiche II7).

16 Cette société de Firminy participe également aux expositions universelles. Voir mon étude sur la participation des facteurs d'instruments aux expositions nationales et universelles au XIX ${ }^{\mathrm{e}}$ siècle, Malou Haine : Tableaux individuels de la participation des facteurs d'instruments de musique français aux 32 expositions nationales et universelles du XIX ${ }^{\mathrm{e}}$ siècle, publication en ligne (avril 2008), www.iremus.cnrs. $\mathrm{fr} /$ sites/default/files/expositions_I798-I900.pdf(25 juin 20I8). 
Déposants issus d'autres villes que Paris La majorité des marques étant déposées au greffe du Tribunal de Commerce de Paris, il va de soi que ce sont principalement des luthiers, facteurs ou marchands parisiens ou installés à Paris qui vont y faire enregistrer leurs marques. Or, les Archives de Paris conservent aussi un certain nombre de marques déposées au greffe de tribunaux de commerce d'autres villes: aucune distinction de classement ne les différencie des marques déposées par des Parisiens, pas plus que les marques étrangères évoquées ci-après, si ce n'est quelquefois l'indication manuscrite de « duplicata », ajoutée sur le document déposé. C'est donc la lecture des fiches qui précise de quel tribunal de commerce elles proviennent (voir le sixième champ de chaque fiche). Nombreux sont les facteurs installés en province qui disposent d'une succursale à Paris où ils déposent leurs marques. Certains facteurs ou marchands ne semblent pas avoir de dépôt à Paris, mais leurs marques sont également déposées au greffe du Tribunal de Commerce de Paris.

L'index des villes recense des luthiers et facteurs d'instruments installés dans quelque soixante-dix villes de France : de Lille à Marseille et de Mirecourt à Albi en passant par Firminy, Jenzat, Tours, Le Puy ou Grenoble, pour n'en citer que quelques-unes. La plupart du temps, ces fabricants font appel à des mandataires pour déposer leurs marques, mais il n'est pas non plus inhabituel qu'ils les déposent eux-mêmes directement : J. B. Blanche-Petit, facteur de pianos automatiques de Valenciennes, dépose luimême ses marques en I9I9 (fiches II25 et II26).

Déposants étrangers Selon la loi du 23juin I857, les étrangers qui possèdent en France des succursales peuvent bénéficier de cette loi. Il en va de même pour les Français dont les établissements sont situés hors de France, à condition que les pays où ils sont installés aient établi un traité de réciprocité en la matière. Dans ce cas, le dépôt des marques étrangères se fait au greffe du Tribunal de Commerce de la Seine, c'est-à-dire à Paris.

Les marques enregistrées d'abord à l'étranger avant d'être (re)déposée à Paris par un mandataire sont assez abondantes et représentent un peu plus de ro \% de l'ensemble des marques recensées : I46 marques déposées par 90 facteurs ou firmes issus de dix pays différents. L'Allemagne-Autriche vient en tête avec 6I marques pour 32 déposants, suivie des États-Unis (4I marques pour 22 déposants) et de l'Angleterre (2I marques pour I8 déposants). Les autres pays, tels l'Italie, la Belgique, la Suisse, le Canada, l'Espagne, les Pays-Bas et la Suède sont peu représentés, avec moins de cinq marques chacun (voir le tableau ci-dessous qui détaille également les villes représentées).

17 Voir Pauline Simons: Le piano avant-gardiste de Pleyel, in : Le Figaro, 27 septembre 20I2, www.le figaro.fr/musique/2012/09/27/03006-20120927ARTFIGo0387-le-piano-avant-gardiste-de-pleyel.php. 
Pays Villes*

Deux chiffres suivent les noms des villes : le premier représente le nombre de déposants ; le second, mentionné entre parenthèses, précise le nombre de marques déposées. Ainsi, «Berlin 3 (7) » signifie que trois facteurs ou firmes ont déposé sept marques. Voir l'index des villes pour le détail des noms.

Allemagne- Berlin 3 (7) / Bockenheim ${ }^{* *}$ I (I) / Böhlitz I (I) / BrunndobAutriche $\mathrm{ra}^{* \star *}$ I (2) / Dresde I (2) / Eisenberg I (I) / Francfort I (4) / Freiburg I (I) / Gera I (I) / Gohlis I (I) / Hanovre I (I) / Iserlohn 2 (2) / Klingenthal 3 (3) / Leipzig 5 (I4) / Markneukirchen 3 (8) / Mülheim 2 (4) / Offenbach s/Main I (I) / Trossingen I (3) / Vienne I (I) / Wahren ${ }^{\star \star \star *}$ I (2)

États-Unis Boston I (I) / Chicago 2 (3) / Cincinnati I (I) / Columbia 2 (5) / Manhattan I (I) / New York Io (25) / Ohio I (I) / Philadelphie I (I) / Rochester I (I) / Saint-Louis I (I) / Worcester I (I)

Angleterre Hackney ${ }^{* \star * * *}$ I (I) / Hayes I (I) / Leigh on Sea I (I) / London I3 (I6) / Steyning I (I) / Warrington I (I)

Italie Bologne I (I) / Crémone I (I) / Milan 2 (2) / Naples I (I) / Rome I (I)

Belgique Bruxelles 3(3)

Suisse La Chaux-de-Fonds 3 (3) / Sainte-Croix I (2)

Canada Montréal 2 (4)

Espagne Barcelone 2 (2) / Madrid I (I)

Pays-Bas La Haye I (I)

Suède Stockholm I (I)

Total 3

Total des Total des marques déposants déposées

* Les noms des villes sont indiqués selon l'orthographe utilisée par les déposants, tantôt dans la langue originale, tantôt dans la version française.

** Aujourd'hui rattaché à la ville de Francfort.

*** Recte : Brunndöbra, aujourd'hui rattaché à Klingenthal.

$\star \star \star *$ Aujourd'hui rattaché à la ville de Leipzig.

$\star * \star * \star$ Aujourd'hui rattaché à la ville de Londres. 
Si plusieurs firmes étrangères disposent de succursales ou de représentants à Paris, si plusieurs facteurs ont racheté ou acquis par fusion diverses marques, d'autres en revanche déposent leurs marques à Paris par l'intermédiaire de mandataires sans que l'on sache précisément le nom du représentant de ces marques dans la capitale.

Les adresses fournies par certaines firmes permettent immédiatement d'apprécier leur succès et leur développement. C'est le cas notamment de «The Aeolian Compagny » dont le siège est situé sur la Cinquième Avenue de New York (pour six marques déposées entre janvier I9OI et décembre I909), puis, à partir de janvier I9II, elle dispose d'une succursale Avenue de l'Opéra à Paris.

Contenu et caractéristiques des marques de la facture instrumentale La matérialité de la marque et son procédé d'application peuvent prendre plusieurs formes : étiquette manuscrite ou pré-imprimée à coller dans des violons ou sur des boîtes et paquets d'emballage, marque en creux à appliquer à l'aide d'un poinçon (sur des pavillons, becs de clarinette, etc.), empreinte à sec ou à chaud à apposer avec un poinçon, marque à imprimer sur des documents administratifs (papier à en-tête, factures, etc.). Rares sont les marques de grandeur réelle, car les déposants se réservent le droit de les reproduire dans toutes les dimensions. Pourtant, quelques-uns spécifient les dimensions exactes de leurs marques : Paquet et Fils précisent que la lyre lui servant de logo doit avoir « $55 \mathrm{~mm}$ de haut sur $32 \mathrm{~mm}$ de large » (fiche 84); Jean Albert Bernard indique que la marque nominative « Paolo Amiati / Paris » a pour dimensions «2I, $5 \mathrm{~cm}$ de long sur $6 \mathrm{~cm}$ de large » (fiche IOo).

Rappelons que déposer sa marque n'est pas un acte administratif obligatoire. Ainsi trouve-t-on un facteur renommé comme Adolphe Sax dont la marque n'a jamais été enregistrée de son vivant, mais elle le sera par ses successeurs, la maison Selmer. Le facteur parisien d'instruments à vent François Lefevre ne dépose pas non plus sa marque, mais elle est enregistrée, tour à tour au gré des successions et des rachats, par André Thibouville et $C^{\text {ie }}$ en I892 (fiche I5I), par Désiré Étienne Thibouville en I904 (fiches 396 et 397), par Siéver en I909 (fiche 567), puis par la «Compagnie française des disques et machines Odeon et d'instruments de musique » en I9I9 (fiche II63). Ces passages de marque de firme en firme laissent d'ailleurs supposer que lors de rachats ou de successions, l'ensemble des marques de la maison d'origine ne tombe pas nécessairement toutes dans l'escarcelle du successeur, mais que ce dernier peut très bien à un moment ou à un autre renoncer à l'une ou plusieurs d'entre elles et les céder à son tour. À l'inverse d'un Sax ou d'un Lefevre qui ne voient pas la nécessité de faire enregistrer leurs marques, la maison Jérôme Thibouville-Lamy \& $\mathrm{C}^{\mathrm{ie}}$ dépose plus de soixante-dix marques au cours de la période étudiée. Il est vrai que ce dernier se fait le champion de la distribution à bon marché de tout type d'instruments de musique, non seulement par simple extension de 
sa maison, mais aussi par rachats et fusions diverses, tout en gardant la même raison sociale.

Le contenu des marques varie en fonction non seulement de l'imagination et de l'ego des déposants, mais aussi de leur habileté à utiliser ou à inventer des procédés publicitaires. Car une marque doit susciter l'achat du produit, tant par les éléments informatifs qu'elle comprend (patronyme, lieu d'exploitation, formation de l'artisan, médailles et décorations officielles) que par les éléments suggestifs de qualité (marque avec des noms communs et/ou des éléments figuratifs).

Une marque doit pouvoir se transcender, communiquer une histoire ou une qualité : certaines y parviennent avec succès. Le logo se différencie de la marque par sa représentation graphique. Une marque spécifique peut varier de logo au cours des années, c'est-à-dire modifier sa représentation graphique tant par sa police de caractères que par son contenu. Plus celle-ci est originale et puissante de suggestion, plus s'accroît son efficacité : elle doit pouvoir déclencher l'envie d'acheter. Un logo remarquable et remarqué finit par s'identifier à la marque elle-même et ... devenir un nom commun. On finit par oublier que les termes «Phonographe» ou «Pianola » étaient à l'origine des marques de machines reproductrices de sons avant de désigner des objets spécifiques.

Une marque comprend un ou plusieurs éléments combinés : initiales, patronyme (réel, emprunté ou inventé), signature, nom commun, adjectif qualitatif, mention de villes ou de région, devise, logo, blason, dessin, emblème, enseigne, photo, etc. De simple emblème ou signature à une pléthore de signes divers, les marques des facteurs d'instruments de musique different abondamment les unes des autres. Il est cependant possible d'en dégager des caractéristiques communes.

Initiales comme marques Une marque peut être constituée, en tout ou en partie, d'initiales se rapportant au nom même du fabricant (firme, société, maison, etc.). Dans le cas de rachats de fonds, les initiales originales sont maintenues, mais n'ont alors plus aucun rapport avec la maison qui en détient le monopole : par ex. Arsène Zoë Lecomte dépose une marque en 1860 composée d'un diapason et des initiales de sa maison «A. L. \& C $\mathrm{C}^{\mathrm{ie}}$ » (fiche 2), initiales qui se retrouvent dans une marque qu'il modifie en I87o (fiche 39) et qui sera ensuite enregistrée en 1902 par Couesnon et $C^{\text {ie }}$ (fiches 324 et 325) et enfin par Charles et Jacques Ullmann en I908 (fiche 544).

Lors de fusion, il se peut qu'une marque indique par les initiales utilisées les noms des divers associés qu'elle représente : la marque «PGB » renvoie à Pélisson, Guinot \& Blanchon (fiche 8I6, voir Illustration 3); celle de « S. T. - B. E. » à H. Sabathier, E. Trespaillé-Barrau (fiches 2II et 2I2) avant de passer à la « Société Française des cylindres artistiques pour phonographes \& graphophones» (fiche 239). Les initiales constituent 
parfois des anagrammes partielles : « EVSF » pour Evette et Schaeffer (fiche 900); «Bela. Edya » pour Albert Deblaye (fiche 927).

L'endogamie professionnelle, ou transmission intergénérationnelle d'un même métier au sein d'une même famille, reste assez présente au cours des deux premiers tiers du XIX ${ }^{e}$ siècle. ${ }^{18}$ Une fratrie, voire même tout un ensemble d'oncles et de cousins peut très bien exercer le même métier. Ainsi, les Thibouville, dont une branche exerce dans l'Eure (à La Couture-Boussey et à Ivry-la-Bataille) et à Paris, et dont une autre, les ThibouvilleLamy, sont implantés à la fois à Paris et à Mirecourt, utilisent notamment des initiales différentes pour se distinguer les uns les autres, tout en intégrant d'autres signes distinctifs : Louis Émile Jérôme Thibouville : « L. E. J. T.» (fiche I50); André Thibouville et Cie: « L. T.» (fiche I5I); Jérôme Thibouville-Lamy: «J. T. L.» (fiche 23) et «E» (fiche 56); J. Thibouville Lamy \& C $C^{\text {ie }}$ : «M. F. M. » (fiche 379) et « T. M. » (fiche I027); Martin Thibouville Fils : « T. - M. » (fiche 230).

Notons que des initiales semblables renvoient quelquefois à des noms différents : dans ce cas, c'est souvent la police de caractères qui les différencie, ou d'autres éléments décoratifs composant la marque. Plusieurs cas sont référencés dans l'index des noms. Citons notamment : « A. B. » pour Alphonse Blondel ou Albert Bretonneau ; « A. L. » pour A. Latouches ou Alfred Lejeune; « B. C. » pour Buffet Crampon ou Baudassé-Cazottes ; «F. B.» pour F. Barbier, F. Besson ou François Billaut.

Rares sont les marques constituées d'une seule initiale : «G. » pour Gautrot aîné \& Cie ou Gervex; « L. » pour la Chambre Syndicale des Pianos et Orgues ou pour Lindstrom. Remarquons aussi que plusieurs initiales entrelacées apparaissent dans des monogrammes, accompagnées ou non d'autres ornements complémentaires.

Marques nominatives Les marques nominatives comprennent un patronyme, avec ou sans prénom. Elles se présentent imprimées ou calligraphiées, parfois comme la réplique même d'une signature manuscrite. Des polices de caractères des plus variées fournissent un cachet personnel à ces marques nominatives qui sont le plus souvent utilisées seules, mais parfois aussi accompagnées d'autres signes distinctifs.

Donnons quelques exemples de marques nominatives employées seules ou avec d'autres signes distinctifs : « Cottereau », « Magnière », « Mustel », « Pécatte », « Pleyel», «Schwander », «Steinway». Un seul cas de marque avec un prénom seul a été constaté, celui de «Lucien / Paris» (fiche 372). Les signatures manuscrites sont, elles aussi, assez nombreuses, citons notamment: «L. Blériot», «A. Bosio», «A. Bord», «Brazzi», «Collin-Mézin », « Gélas », « L. Lumière », « Mustel et $C^{\mathrm{ie}}$ », «Pleyel, Wolff \& $\mathrm{C}^{\mathrm{ie}}$ ». Dans 
ces deux derniers cas, c'est la raison sociale, transcrite dans une écriture manuscrite, qui fonctionne comme une authentique signature.

Certains patronymes sont, dans un premier temps, identiques aux noms des déposants des marques. Toutefois, lorsque les fonds de commerce passent dans d'autres mains, voire sortent du milieu familial, ces patronymes n'ont plus aucun lien nominatif avec les déposants.

Certaines marques nominatives dérivent du patronyme du déposant: «Ullmanigraphe» destinée à des phonographes de la Société Ullmann (fiche 224), «Dupinophone » pour des clarinettes fabriquées par Jules Dupin (fiche 243), ou « Didierphone» pour des machines parlantes sans pavillon, de Victor Didier (fiche 690).

Successeurs mentionnés dans les marques Se prévaloir comme successeur d'une maison prestigieuse donne de l'aura au nouveau détendeur de la marque. Pourtant, très peu de luthiers ou facteurs en font mention dans leurs marques. Citons le facteur d'accordéons Paul Ernest Boullay qui s'affiche comme successeur de Georges Kanéguissert (fiche 72).

Lorsqu'il s'agit d'une lignée directe ou connexe, cela semble évidemment moins nécessaire, mais certains l'indiquent cependant, tel Jean-Baptiste Pajot : « Ancienne Maison Pajot Père et Fils / Fondée en I795 / Marque de fabrique déposée/ Pajot Fils seul suc ${ }^{\text {r }}$ Jenzat(Allier)» (fiche I04). Charles Chipot-Vuillaume rappelle qu'il est le gendre de JeanBaptiste Vuillaume (fiche I28).

Les successions se mentionnent de préférence dans la case réservée au nom du déposant et non dans celle de la marque : Caressa et Français se disent successeurs de Gustave Bernardel lorsqu'ils redéposent la marque de celui-ci (fiches 997 et 998). Le fabricant de boîtes à musique Henri Maire s'affiche comme le successeur de H. Suttner (fiche I050). Henry Chéry Selmer mentionne qu'il est le successeur de Florentin Barbier lorsque la marque de ce dernier est redéposée (fiche II22). Evette \& Schaeffer s'affichent comme les successeurs de Paul Goumas dans plusieurs de leurs marques (voir index des noms).

Les cas les plus nombreux portent sur des déposants qui font enregistrer des marques connues dont les patronymes ne sont pas les leurs, ce qui sous-entend évidemment qu'ils en sont les dépositaires, sinon ils s'exposeraient à des poursuites judiciaires. L'index des noms permet de constater les multiples marques rachetées par Couesnon, ThibouvilleLamy ou encore Ullmann, à côté de patronymes fictifs également utilisés par ces diverses maisons.

Affiliations et formations précisées dans les marques Se prévaloir d'une formation effectuée auprès d'un maître luthier suggère évidemment un travail soigné de grande 
qualité. Ainsi, Léon Mougenot se présente comme un « ex-ouvrier » des premières maisons de Bruxelles, Lyon, Paris et Londres (fiche 889, voir Illustration 4). Théophile Merz se dit ancien élève d'Eugène Gand (fiche 257). Lorsque la société Ullmann dépose la marque du luthier Paul Bailly qu'il rachète en I9ıo, elle précise que ce dernier fut un élève de Jean-Baptiste Vuillaume (fiche 669).

Noms de virtuoses utilisés comme marques Utiliser des noms de virtuoses pour dénommer certains instruments ou accessoires, c'est évoquer les qualités d'excellence de ces interprètes et laisser croire, de manière inconsciente, que celles-ci se sont reportées sur les produits concernés ou que ceux qui les utiliseront parviendront à égaler les artistes cités.

Charles Ullmann a choisi la marque « VIOTTI » (fiche 398) pour ses violons et cordes harmoniques. Couesnon \& $\mathrm{C}^{\text {ie }}$ appliquent l'expression «Véritable PAGANINI » à leurs chanterelles (fiche 339). Adam Morhange recourt lui aussi au célèbre virtuose pour ses «CHANTERELles PAGANINI » et renforce encore l'illusion en l'accompagnant de son portrait (fiche 543). Édouard de Cristofaro a choisi un autre grand interprète du violon pour qualifier les cordes qu'il met en vente : «LA WIENIAwSKI » (fiche 700, voir Illustration 5). Les frères Schuster, de Markneukirchen en Allemagne, utilisent « TARTINI » pour faire valoir leurs instruments (fiche 639). Le négociant Charles Morice utilise le nom d'un pianiste célèbre à l'époque (et encore vivant au moment de l'exploitation) pour vendre ses pianos et harmoniums : «Diémer \& Cie » (fiche 208), sans doute avec des compensations financières pour ce dernier.

Ce procédé métaphorique établit une correspondance entre le personnage évoqué et le produit vendu, allant même jusqu'à la personnification puisqu'il peut représenter un produit sous les traits d'un individu. Ce subterfuge publicitaire, naïf mais très efficace, ne trompe cependant personne. Il en va tout autrement de l'emploi de patronymes fictifs utilisés afin de tromper volontairement le client (voir plus avant « Marques trompeuses »).

Noms d'instruments utilisés comme marques Une marque peut aussi s'identifier complètement au nom de l'instrument nouvellement inventé par son déposant. Celui-ci ajoute d'ailleurs souvent la mention «breveté » dans sa marque (voir ci-dessous). Mais déposer une marque qui s'identifie au nom d'un instrument nouveau évite aussi, dans certains cas, l'enregistrement d'un brevet plus coûteux.

Un très grand nombre de marques de ce type ont été recensées. Sans pour autant être exhaustive, signalons «Arionette » (fiche 309), «Autoharp » (fiche 55), «Clavitube » (fiche 207), « Cornon » (fiche I29), « Cornophon » (fiche I30), « Cornophones » (fiche 4II), «Le Dupinophone » (fiche 243), « Gavioliphone » (fiche 352), « Guide main W. Bohrer » 
(fiche 43), « Main doublée » (fiche 89), « Mignon » (fiche 406), « Mélotétraphone » (fiche I59), «Molliphone» (fiche II4), «Musicographe » (fiche 247), « Ocariflûte » (fiche 289), «Orchestrophone» (fiche 206), «Phonola (fiche 402), «Piano eutonophone», (fiche II8), «Pianola» (fiche 310), «Pleyela (fiche 393), «Polyphon» (fiches 234 et 303), «Le Pyrophone » (fiche 6I), «Vendoline » (fiche 30I) et « Violaline » (fiche 264). ${ }^{\text {I9 }}$

Il arrive aussi quelquefois qu'un nom d'instrument soit donné comme marque alors que sa destination ne correspond nullement au nom utilisé, par exemple la marque «Célesta» destinée aux cordes pour mandolines et non à l'instrument à percussion (fiche 884); «Cloche» s'applique à des cordes harmoniques (fiche 607); «Mandoliphone » est affectée aux petits pianos munis de marteaux trembleurs et non aux mandolines (fiche II3); «A la Cymbale» désigne des cordes harmoniques (fiche 350); citons encore la marque banale de « Pianos » qui s'identifie à des accordéons (fiche 348).

Mention des brevets Un facteur qui a déposé un brevet pour une invention ou un perfectionnement souhaitera parfois le mentionner dans sa marque, donnant ici aussi l'illusion d'une reconnaissance officielle : or l'on sait que les brevets constituent de simples enregistrements, tout comme les marques de fabrique. Le gouvernement oblige d'ailleurs à mentionner le sigle « s.G.D.G. », ce qui signifie « sans garantie du gouvernement » lorsque le terme brevet ou breveté est cité. Utiliser le sigle seul laisse planer un parfum de mystère sur le produit, ce qui accrô̂t sa puissance évocatrice, alors que sa signification même est réductrice.

Certains respectent cette règle, tels Djalma Julliot (fiche I69), François Joseph Tessereau (fiche I9), Émile Mennesson (fiche 47) ou Alphonse Blondel (fiche 54), pour n'en citer que quelques-uns qui affichent «breveté s.G.D.G. ». Ils la respectent d'autant plus lorsque les noms des inventions figurent aussi dans la marque, comme la lyre Véga de Jean Joseph Roche (fiche 548), la mécanique à bayonnettes [sic] Molliphone d'Émile Mennesson (fiche II4), ou encore le piano Eutophone d'Émile Legay (fiche Iı8).

En revanche, d'autres ignorent cette réglementation et se contentent d'un simple «breveté », comme Auguste Courtois aîné (fiche 3), Jérôme Thibouville-Lamy (fiche 23) ou Charles Collin-Mézin (fiche II2I). Jean Schwander et Joseph Heerburger (fiche 55) préferent signaler qu'ils disposent de «brevets en Europe \& en Amérique ».

Par prudence ou par malice peut-être, un seul facteur dépose deux marques différentes le même jour, l'une avec la mention s.G.D.G., l'autre pas. Il s'agit de Gautrot aîné, avec la marque « Gautrot-Marquet / Breveté S.G.D.G. / Paris » (fiche 28) et celle de « Gautrot Breveté / à Paris » (fiche 29). 
Mention ou évocation d'une ville, d'une région, d'un département Plusieurs luthiers ou facteurs d'instruments désirent affirmer leur attachement à leur ville, leur région ou leur département en le précisant dans leurs marques, soit par le nom même (Dijon, Lyon, Ardennes, Champagne, etc.), soit par un adjectif dérivé (champenois, verdinois, vénitien, etc.). Plusieurs villes sont citées en latin, choix effectué par les fabricants dans un souci d'élégance et d'élitisme: Avenio (Avignon), Nicæa (Nice), Massillia (Marseille), Lutetia (Paris), etc. D'autres préferent citer des villes en italien ou en espagnol afin de faire croire que leurs instruments sont originaires d'Italie ou d'Espagne (voir plus avant « marques trompeuses »). Un cours d'eau peut vouloir situer une région, telle la marque nominative de cette «Usine hydraulique des forces motrices du Madon», que l'on devine située en Lorraine. L'appartenance à une ville (à une région ou à un département) s'exprime plus fortement encore lorsque celle-ci figure dans la raison sociale de la maison reprise dans la marque nominative: «Aciéries et Forges de Firminy» ou «Forges de Châtillon et Commentry ». La « Société niçoise de Pianos Automatiques » souligne son lieu d'exploitation par la marque « Piano Nicæa » (fiche 679).

L'évocation d'une ville ou d'une région peut être symbolisée par une expression, un dessin ou une photographie. Émile Mennesson, installé à Reims, utilise la marque «Le grand Bailla » (fiche 64, voir Illustration 6), nom du dragon que l'on promène dans les rues de la ville une fois par an, rappelant la tradition moyenâgeuse de la région. BaudasséCazotte, fabricant de cordes harmoniques installé à Montpellier, affiche une photographie de l'esplanade du Peyrou, avec son château d'eau monumental et la statue équestre de Louis XIV (fiche I4). Ferdinand Monti, spécialisé dans les fournitures de pianos, se sert de l'éléphant de Barbizon, signifiant ainsi sa présence dans la région, plus précisément à Champigny sur Marne (fiche 40). Non content de représenter le blason de la ville de Mirecourt, le luthier Gabriel Didion indique sous celui-ci «A la ville de Mirecourt ». Le chardon lorrain fait partie de la marque des établissements Laberte-Humbert Frères \& Fourier Magnié réunis établis eux aussi à Mirecourt (fiche II46). ${ }^{20}$

À l'inverse, plusieurs facteurs de province indiquent le nom de la capitale française dans leurs marques afin de faire croire qu'ils sont les dépositaires d'instruments parisiens. C'est le cas notamment de Paul Cestin à Alger, Émile Pouzol à Avignon, A. Laput à Clermont-Ferrand, Raver ou André Delmouly à Bordeaux, Émile Grunig à Grenoble, A. Rabut à Lyon, Henri Graffan à Marseille et Charles Morice à Rennes.

Noms de villes étrangères suite à des fusions, rachats, importations, etc. Il se peut aussi que l'emploi d'une marque fasse suite à une association ou à un rachat. C'est le cas, déjà mentionné, de Derazey qui utilise la marque d'une maison rachetée. 
Par ailleurs, la mention par un fabricant français d'une ville étrangère peut simplement vouloir dire qu'il importe le produit concerné. Les luthiers Maxime Fabre de Valenciennes (fiche 9I5) et Julien Lavest de Montluçon (fiche IO3I) utilisent les mentions respectives de «INSEnsible / Corde HONGRoise ... » et «LA HONGroise / Nouveau procédé KEMÉNY » pour vendre des cordes de violon.

De même, Marc Laberte (fiche 824) à Mirecourt utilise la marque « CORDE PADOVA Importée CANTA BENE » pour distinguer ses instruments de musique et accessoires. Paul Imbert se sert des marques «Les célèbres cordes AUSTRIA » (fiche IOI5) ou « Les célèbres cordes ITALIA » (fiche IOI4), rappelant ainsi une époque où les cordes venues d'Autriche et d'Italie étaient considérées comme les meilleures d'Europe. Toutefois, on peut se demander si ce luthier importe vraiment ces cordes ou s'il use d'un procédé publicitaire qui en donne l'illusion, car certains noms de villes sont utilisés à dessein afin de tromper le client (voir plus avant).

Des qualités utilisées comme marques Ce sont surtout les fabricants ou vendeurs de cordes qui utilisent des marques qualitatives, mais d'autres artisans ou industriels ont aussi recours à ces appellations efficaces qui touchent l'inconscient. Bien avant les travaux des neurosciences cognitives appliquées au marketing (neuromarketing) du début du XXI ${ }^{e}$ siècle, les artisans et industriels du XIX ${ }^{e}$ siècle avaient trouvé des procédés publicitaires puissants pour attirer des clients.

Des appellations telles «L'Universelle» pour des cordes harmoniques (fiche 312), «La Divina » pour des mandolines (fiche 33I) ou « Parfait» pour des pianos (fiche 338) constituent autant de stimuli positifs auprès d'une clientèle qui leur préférera ces marques à celles plus banales de « chanterelles pour violons », « Gut Silk», « Harmonic » ou « Paris-pianos ». Comment résister à ces marques qualitatives dont la puissance évocatrice est évidente : «L'Apothéose » (fiche 6II), «Le Bijou » (fiche 346), «L'Incroyable » (fiche 705), «L'Invincible » (fiche 836), «La Mélodieuse » (fiche 247), «La Merveilleuse » (fiche 240), « La Résistante » (fiche 280), « La Séduisante » (fiche 6I2), « La Sonora » (fiche 277), « La Suave incassable » (fiche 384), « La Transparente » (fiche 63), « Le Triomphant » (fiche 342), « La Victoriosa » (fiche 337), etc.

Les métaphores d'excellence produisent les mêmes effets suggestifs avec des termes comme «Apollon» (fiche 490), «Eden» (fiche 395), « Hercule» (fiche I96), «Phébus » (fiche I92), «Phénix » (fiche 420) ou «Vénus » (fiche 982). On préfere ici le nom même de certains dieux, plutôt que leur représentation illustrée. La qualité supposée est parfois plus directement présente lorsque celle-ci s'intitule tout simplement « $\mathrm{Q}^{\text {té }}$ extra » [pour Qualité Extra] (fiche II), «Qualité Excelsior» (fiche 275) ou «Qualité supérieure» (fiche 47). Reconnaissons toutefois que la suggestion s'avère ici beaucoup moins poétique : le vraisemblable fait rêver, tandis qu'une affirmation péremptoire fait sourire ... 
Charles Cahit tire parti de son patronyme pour déposer une marque intitulée « Le Cahit dit le merveilleux », jouant ainsi avec l'homophonie du mot « caïd» (fiche 330).

Médailles d'exposition et autres reconnaissances officielles L'excellence peut être suggérée par une couronne de laurier (fiche 157) ou par des médailles remportées lors des expositions universelles ou des expositions régionales de produits de l'industrie. Puisque ces médailles sont attribuées par un jury, elles sont censées cautionner objectivement la valeur de ceux qui les reçoivent. ${ }^{2 \mathrm{I}}$ Après avoir obtenu deux médailles d'argent, respectivement en I878 et I889, le luthier Charles Collin-Mézin (fiche II07) est fier d'afficher sur ses instruments son « Grand Prix » de l'Exposition Universelle de I9oo.

Lorsque ces médailles sont trop nombreuses, on ne les cite plus, mais on les représente par des photographies. Certains n'hésitent d'ailleurs pas à donner l'illusion d'en avoir remporté un très grand nombre : Thibouville-Lamy fait représenter seize médailles, alors qu'il n'en a gagné que huit au moment du dépôt de sa marque en I866, mais elles s'affichent recto verso (fiche I2). Joseph Baudassé agit de même (fiche I4). Se trouver hors-concours ou membre du jury lors de ces expositions confere évidemment une excellence supérieure aux autres : les marques d'Alexandre Mangenot (fiche 263), Couesnon \& C (ie (fiche 326) ou Thibouville-Lamy (fiche 300) en font état.

Lors de rachats ou successions, la maison héritière dispose du droit de se vanter des médailles obtenues par son prédécesseur. Ainsi, Paul Pélicier pourra afficher sa marque « Médailles d'Or / Pélicier / Paris » (fiche 97), alors que c'est en fait Georges Bachmann qui a remporté ces prix et médailles.

La vanité de certains facteurs les pousse à mentionner aussi les reconnaissances et distinctions officielles qu'ils ont obtenues. Jérôme Thibouville-Lamy précise qu'il est breveté, Officier de la Légion d'Honneur et Chevalier de l'Ordre de François-Joseph (fiche 300). Plus les reconnaissances officielles seront nombreuses, plus importante sera l'excellence supposée des instruments fabriqués.

Proverbes, devises, maximes, etc. Les luthiers et facteurs d'instruments utilisent peu de proverbes, devises, adages, axiomes et autres formules dans leurs marques. Seuls cinq cas répondent à ce critère : Édouard Joseph Mangeot applique deux expressions dans une même marque : «Devines [sic] si tu peux » et « Fais-le si tu oses », variante d'une citation

Voir nos travaux sur les facteurs français récompensés aux expositions nationales et universelles entre I789 et I900, notamment les Tableaux individuels de la participation des facteurs d'instruments de musique français aux 32 expositions nationales et universelles du xIxe siècle, www.iremus.cnrs.fr/sites/default/files/ expositions_I798-I900.pdf. 
bien connue de l'Héraclius de Pierre Corneille. ${ }^{22}$ Ce facteur lance ainsi un défi à quiconque s'aventurerait à copier ses « pianos suédois » (fiche 79, voir Illustration 7). Quant à Jérôme Thibouville Lamy \& $C^{\text {ie }}$, c'est la formule latine «In aqua vis », sans autre signe distinctif, qui lui sert de marque en février I903 (fiche 358). Sans doute ses clients ont-ils trouvé celle-ci trop énigmatique, car neuf mois plus tard, il fait enregistrer une nouvelle marque, comprenant toujours cette expression latine, mais agrémentée cette fois d'un dessin représentant une roue à aube, ce qui rend la formulation plus explicite (fiche 379). Ce même facteur prend aussi la devise « Dulcis et Fortis » pour logo à appliquer sur ses flûtes et clarinettes (fiche I08). La Compagnie Générale de Cinématographes, Phonographes et Pellicules fait dire à un coq triomphant: «Je chante haut et clair», devise destinée aux phonographes et gramophones (fiche 2I5). Enfin, « SÉ CANTO, QUÉ CANTO » s'applique à tout type d'instrument de musique par Boskamp \& Cie (fiche 96I).

Nombres écrits en chiffres Rares sont les marques constituées d'un nombre écrit en chiffres, à l'exclusion de toute autre marque distinctive. La société Ullmann, qui fabrique plusieurs catégories d'instruments de musique, emploie les nombres de 8 à I3, et 4I à 43, pour distinguer ses flûtes (fiches I76 à I8I ; I97 à I99). Maurice Schwob emploie celui de I00 000 qui peut, précise-t-il, s'écrire en chiffres ou en lettres (fiche 458) : laisse-t-il supposer qu'il a vendu un aussi grand nombre d'instruments, suggérant ainsi leur succès ?

Éléments figuratifs Outre des mentions de noms, d'instruments, de villes, de qualités, etc., la plupart des marques comprennent également de nombreux éléments figuratifs : notes et portées musicales, emblèmes, blasons, dessins, photos, etc. Ces éléments figuratifs ont un pouvoir de suggestion plus important encore que le texte même d'une marque, puisqu'il agit sur l'inconscient : c'est là une pratique commerciale bien connue.

Quelques éléments figuratifs ont déjà été évoqués ci-dessus, notamment les médailles d'exposition remportées par les déposants, ou des allusions picturales permettant de situer le lieu d'exploitation (éléphant de Barbizon, esplanade de Montpellier, chardon lorrain ou blasons divers).

Sans surprise, les instruments de musique constituent les éléments figuratifs le plus souvent représentés, ${ }^{23}$ et plus particulièrement lorsqu'il s'agit d'inventions spécifiques : arionette, autoharp, babouches-grelots, etc. Pas étonnant non plus si un dessin ou une photographie d'un instrument spécifique ou d'un accessoire est précisément destiné à être utilisé comme marque. Ainsi, une représentation de violon est forcément à utiliser

«Devine, si tu peux, \& choisy, si tu l'oses. » Pierre Corneille : Héraclius, Paris I653 ('I647), p. 57 (Acte IV, Scène v).

23 Voir le recensement complet dans l'index des éléments figuratifs. 
sur des violons, celle d'un bec de clarinette à des anches de clarinette, celle d'un phonographe pour des machines parlantes. Seul Baudassé-Cazottes fournit une vue d'ensemble de ses ateliers (fiche 4I). Plus banales sont ces silhouettes de personnages jouant d'un instrument.

Mais des instruments ou accessoires symboliques, tels le diapason ou la lyre, sont souvent présents afin d'évoquer la musique en général : ces symboles sont alors entourés d'éléments décoratifs, notamment des étoiles. Il en va de même des notes de musique, des portées musicales, des partitions ou encore des clefs de sol ou de fa. Dans le même ordre d'idées, le terme «Euterpia» ou la représentation de personnages mythologiques remplissent le même rôle symbolique : Orphée et sa lyre, une muse à la harpe ou à la lyre.

Certains instruments de musique sont sublimés par des portraits en rapport étroit avec la catégorie concernée : Nicolas Lupot ou Niccolò Paganini pour des violons, Aristide Bruant pour des disques et cylindres de machines parlantes. Les photographies de l'Opéra de Paris, de l'Odéon ou du Trocadéro évoquent à la fois la ville du déposant et les lieux de spectacles où l'on entend de la musique. Le neuromarketing nous apprend que les acheteurs potentiels trouveront ces produits de meilleure qualité parce qu'ils auront été sublimés. Mais quel intérêt de déposer une marque «Corde-Gounod» avec le portrait de ce compositeur (fiche I048, voir Illustration 8) ? Bien que l'on puisse relier le doux sentiment des mélodies de Gounod au son d'un violon, la relation subliminale ici est ratée. On ne comprend pas non plus la raison pour laquelle Theodor Stark, de Markneukirchen en Allemagne, choisit en I9I2 la marque « Rizal», accompagnée du portrait du poète engagé José Rizal (I86I-I896), ce héros national philippin fusillé qui avait visité l'Allemagne juste avant son exécution par l'autorité espagnole, si ce n'est par admiration toute personnelle pour cette personnalité hors du commun. ${ }^{24}$ Ici non plus, pas d'évocation subliminale.

Des représentations d'animaux animent quelquefois les marques des luthiers et facteurs d'instruments. Le coq figure en bonne place parmi les animaux les plus représentés : il symbolise à la fois la France, la victoire et celui qui chante de bonne heure. $\mathrm{Si}$, de plus, il trône sur un globe terrestre, l'impression de supériorité est totale. Une lyre ou une clé de sol peuvent accentuer ces intentions. Le lion est tout aussi représenté : il incarne la force, de même que l'aigle qui possède en outre le prestige. De son côté, la colombe personnifie la douceur, et le cygne l'élégance. On attribue à l'abeille un rôle initiatique : de plus, c'est le symbole de la vertu et de l'éloquence. La chauve-souris qui vit dans le noir est utilisée pour des machines parlantes, disques et cinématographes,

24 S'agirait-il d'un lieu géographique en relation avec cette firme ? Il existe bien une rue José Rizal à Markneukirchen, mais encore faudrait-il qu'elle existât déjà en I9ı2. La Place José Rizal dans le $9^{\mathrm{e}}$ arrondissement de Paris n'a, pour sa part, été inaugurée qu'en octobre 20 II. 
tandis que la mouche ne doit sa présence qu'au patronyme du déposant, Georges Lamouche (fiche 580).

Parmi les corps célestes, l'étoile à cinq branches est la plus représentée, car elle symbolise l'excellence et la lumière. En revanche, le soleil, symbole fort s'il en est, est très rarement exploité. Le croissant de lune s'applique à des cymbales afin de rappeler son origine turque. Un ciel menaçant avec des éclairs se déploie dans la marque du phonographe «Tonnerr [sic]», logo curieux dont la connotation négative ne semble pas appropriée, à moins que ce symbole ne cherche à suggérer la puissance du son.

Les fleurs et végétaux seuls sont relativement rares (quelques couronnes de laurier), de même que les formes géométriques (triangles isocèles). En revanche, on les retrouve en abondance dans les encadrements ornés dans lesquels s'inscrivent les marques et logos. Enfin, quelques éléments figuratifs isolés sont regroupés dans l'index sous « objets divers » (ancre marine, fils, bateau, etc.).

Marques trompeuses Les marques trompeuses sont celles qui comprennent un certain nombre d'informations destinées à faire illusion auprès des clients potentiels : mentions de villes sans rapport avec les activités réelles des déposants et patronymes fictifs utilisés comme pseudonymes. Ces pratiques courantes constituent des astuces publicitaires de sublimation, mais frisent le plus souvent la tromperie. Très rares sont ceux qui avouent la supercherie de ces patronymes utilisés comme pseudonymes (voir plus avant).

Certains fabricants ou marchands mentionnent des noms de villes dans leurs marques pour appâter le client et faire illusion ; or la ville citée n'a souvent rien à voir avec le produit, sauf s'il est en effet véritablement importé : la frontière est évidemment ténue et pas facile à déceler. Ainsi, certains luthiers français indiquent volontiers un nom de ville italienne afin de faire croire que les violons qui sortent de leurs ateliers proviennent d'Italie, garantie d'un savoir-faire ancestral.

Prenons la ville de Crémone qui, dans l'index des villes, comprend quatre facteurs. Seule la firme Rulli Sonori Traforati (fiche 848) y est réellement installée, les autres citent cette ville comme faire-valoir.

La ville de Naples apparaît comme l'abus par excellence de la mystification. Treize luthiers fabriquant des mandolines y sont recensés dans l'index des villes, mais douze d'entre eux sont originaires de France, Algérie comprise évidemment à l'époque : Alfred Bidet (Alger), Georges Carcassonne (Paris), Paul Cestin (Alger), C Ce Française des Disques \& Machines Odéon (Paris), Albert Deblaye (Mirecourt), Maxime Fabre (Valenciennes), Joseph Jules Köchly (Bourges), les frères Laberte-Humbert (Mirecourt), Émile Pouzol (Avignon), Jérôme Thibouville-Lamy (Paris) et Jacques Ullmann (Paris). Leurs marques, pour la plupart des étiquettes, comprennent le nom de la ville de Naples, avec même une nette préférence pour sa formulation italienne « Napoli ». 
À celle-ci sont quasiment toujours associés des prénoms et patronymes aux consonances italiennes, noms fictifs qui ne figurent que rarement dans les ouvrages de référence. ${ }^{25}$ Édouard de Cristofaro est réellement italien : la fabrication des instruments à cordes se fait à Napoli (là où son père exerçait ${ }^{26}$ ), et la vente à Paris. Sa marque est enregistrée au greffe de Paris, mais ne provient pas de Naples. Quant au luthier Giovanni Racca, il exerce réellement à Bologne (fiche 298).

On constatera aussi l'emploi de certains noms dont la consonance flirte dangereusement avec de vrais noms de luthiers anciens. Et pour que l'illusion soit totale, les étiquettes sont souvent rédigées en italien ou en latin. Prenons quelques exemples : LaberteHumbert Frères déposent la marque « Fabricatori d'Instrumenti di Musica/ LUIGI URIANI / NAPOLI / Provedittore di primi Artisti et Professori » (fiche I52), tandis que le facteur de pianos Jean Albert Bernard, de Bordeaux, applique celle de «Paolo Amiati / Paris » (fiche roo).

Charles et Jacques Ullmann, installés à Paris, fabriquent entre autres instruments des guitares et des mandolines sur lesquelles ils appliquent une marque nominative, «Juan Jose Alcofar», suivie du mot «Madrid» (fiche 274) ou celle de « Hijos de Ramon de Redueyo » suivie de la ville «Valencia» (fiche 273). Ces noms de luthiers espagnols sont des patronymes fictifs, inventés afin de servir de pseudonymes : les Ullmann font ainsi croire à leurs clients qu'ils ont racheté des marques espagnoles existantes. De même, Jérôme Thibouville-Lamy emploie le nom de « Luigi Euvingino » sur ses mandolines.

Remarquons que la plupart de ces noms (Barberini, Luigi Dorigo, Gardarni, Guarini, Luigi Marini, Antonio Mazarelli, Alfredi Noluoca, A. Torelli, Luigi Uriani, etc.) se retrouvent aujourd'hui sur des sites de vente en ligne comme ebay ou autres : les instruments portant ces noms fictifs sont renseignés comme des « instruments anciens » d'origine italienne ou espagnole, sans le moindre discernement d'authenticité. La tromperie des luthiers du siècle passé continue d'exercer ses ravages ...

Mais ne condamnons pas en bloc l'ensemble des luthiers dont les étiquettes résonnent aux noms italiens; certains noms sont sans doute de véritables luthiers italiens représentés en France.

Une marque intitulée "Coq gaulois» est appliquée sur des harmonicas importés d'Allemagne (fabriqués par Matthieu Hohner, de Trossingen) de manière à faire croire que ces instruments sont des produits nationaux. Pour renforcer le message, la

25 Giuseppe Tolino est cité dans Vannes: Dictionnaire des luthiers, p.364 pour une «jolie mandoline avec marqueterie ». Il est cependant qualifié de «luthier italien du XIX ${ }^{\mathrm{e}}$ siècle ».

26 Vannes consacre deux courtes notices aux « Cristofaro », l'un à l'Italien, l'autre à son fils E. [Édouard] qui installa la succursale à Paris en I892. La marque ici recensée date de I909, ibid., p.70. 
mention « Harmonica National » figure également dans la marque (fiche 564, voir Illustration 9).

L'aveu de l'emploi d'un patronyme comme pseudonyme n'est mentionné qu'à deux reprises par les déposants au cours de la période étudiée. L'éditeur de musique lyonnais Étienne Rey fils aîné vend des pianos marqués « $\mathrm{H}$. France \& $\mathrm{C}^{\mathrm{O}}$ » afin, écrit-il sur la fiche d'enregistrement, d'en « indiquer la qualité et la provenance » (fiche III). Cette première marque, déposée en décembre I888, est suivie d'une autre quasi identique « H. France», mais le déposant avoue cette fois que la marque est un pseudonyme (fiche II2). Le cas du luthier rémois Émile Mennesson est intéressant. En juin I89I, il dépose une marque avec un patronyme italien dont il est « seul concessionnaire ». On pourrait dès lors en déduire qu'il importe réellement ces instruments d'Italie. Mais avec un patronyme italien et un prénom français («Joseph» et non « Giuseppe »), il n'était guère difficile d'en déduire qu'il utilisait un patronyme comme pseudonyme : «Joseph GUARINI fecit / Anno ... N ${ }^{\circ}$ ... / Emile Mennesson, à Reims (Marne), / Seul concessionnaire pour la France et l'Étranger / DÉPOSÉ » (fiche 22). Dix-sept ans plus tard, ce même luthier dépose à nouveau la marque Guarini, mais avec une modification de taille : « ÉMILE MENNESSON dit GUARINI / Luthier à Ste Cécile à Reims / etc. » (fiche I46). Il ne prétend plus en être le seul concessionnaire, mais ... avoue explicitement que ce patronyme est un pseudonyme : il révèle ainsi au grand public la supercherie. Ce luthier a certainement voulu se démarquer de ses collègues peu scrupuleux. ${ }^{27}$ Notons aussi l'étiquette des frères Tassinari destinée aux mandolines et déposée par la société Ullmann qui, à l’image des luthiers anciens, prévoit d'ajouter une date à la main : toutefois, les trois premiers chiffres de la décennie imprimés (I89 ...) dévoilent que ce luthier se positionne sans tromperie à la fin du xIx $x^{\mathrm{e}}$ siècle, même si la ville «Napoli » laisse croire à des instruments importés (fiche 2I6).

Remarquons qu'une marque comme «A LA VILLE DE CREMONNE [sic]», utilisée par le luthier mirecurtien Derazey après avoir racheté le fonds de Didier Nicolas, n'est pas à considérer comme une tromperie, puisqu'ici la marque s'identifie à l'enseigne de la maison: le nom du fondateur et la mention de la ville de Mirecourt sont également signalés dans la marque (fiches 6 et 70 ).

Les luthiers ne sont pas les seuls à duper leurs clients. Les facteurs de pianos font de même en utilisant des patronymes aux consonances allemandes, puisque la facture d'outre-Rhin est alors gage de qualité, tout comme celle de Paris, à l'origine exercée par une majorité de facteurs allemands expatriés. Ils font ainsi croire que les instruments sont importés ou qu'ils en sont les concessionnaires. L'éditeur de musique bordelais Raver fabrique des pianos frappés « BECKNER à Paris » (fiche 2I): certes, le patronyme présente 
une consonance allemande, mais la mention de la ville de Paris contredit cette suggestion. Raver veut-il alors faire croire qu'il est le dépositaire d'un facteur parisien, lui-même importateur de pianos allemands ou autrichiens ? Un autre facteur de pianos bordelais, André Delmouly, agit exactement de même lorsqu'il utilise la marque « KLEYER / PARIS » (fiche 223). Que dire de ce facteur Paul Cestin, installé à Alger, qui se dit le représentant de « FLAYEL, Paris », flirtant ouvertement avec une petite variante de la célèbre marque parisienne (fiche 320) ?28

Curieusement, un autre facteur bordelais, Jean Albert Bernard, utilise un pseudonyme italien pour ses pianos : « PAOLO AMIATI / Paris »; est-il à ce point ignorant du fait que cette marque ne trompera personne (fiche IOo)?

Une illusion sur des patronymes et/ou les noms de ville ne signifie pas nécessairement des instruments de mauvaise qualité, mais cette pratique s'applique généralement à des instruments fabriqués en masse.

La pratique n'a pas disparu de nos jours. Plusieurs fabricants asiatiques envahissent aujourd'hui les marchés européens avec des marques trompeuses. Il serait d'ailleurs intéressant d'y consacrer une étude approfondie. Contentons-nous de donner deux exemples récents pris dans des domaines très différents, dont l'un n'a rien à voir avec la facture instrumentale. En octobre 20I2, l'emploi de la marque «Laguiole » déposée par un entrepreneur chinois a fait la une des journaux, ${ }^{29}$ interdisant aux couteliers de cette petite ville de l'Aveyron d'utiliser ce nom pour commercialiser leurs produits qu'ils fabriquent d'ailleurs depuis des décennies et qui attirent une clientèle fidèle. Pour marquer sa solidarité avec les couteliers, le maire de Laguiole fit un éclat remarqué en convoquant les journalistes. En présence des couteliers désespérés et furieux, il a déterré le panneau portant le nom de son village : une large bande rouge transversale biffait le nom, signifiant ainsi que le village était rayé de la carte de France.

De même, si la marque «Adolphe Sax» est encore utilisée par la maison Henri Selmer - pratique justifiée par le fait que ce dernier a racheté, le 20 octobre I929, le fonds de commerce de la maison Sax située rue Myrha, 84 à Paris, ${ }^{30}$ Selmer en est donc le successeur légal - il est par contre tout à fait contestable, sur le plan moral, que la marque «Adolphe Sax et $\mathrm{C}^{\mathrm{ie}}$ » soit utilisée par Karel Goetghebeur, ${ }^{3 \mathrm{I}}$ industriel belge installé depuis 2012 à Assebroek en Flandre et qui vend des saxophones fabriqués en Thaïlande.

28 Notons que ce même facteur se dit seul représentant des mandolines signées «V. Gardarini / Napoli » (fiche 32I). Gageons qu'il s'agit certainement d'un marchand et non d'un véritable luthier.

29 Voir notamment La Dépêche du 9 octobre 2012.

30 Malou Haine: Adolphe Sax. Sa vie, son œuvre, ses instruments de musique, Bruxelles I980, p. I35.

31 Marque «Adolphe Sax \& Cie » déposée par Karel Goetghebeur à l'Institut de la Propriété industrielle de Paris le 27 février 2012 pour une période de dix ans. 
Cet usage est d'autant plus abusif que le site «Adolphe Sax et $\mathrm{C}^{\text {ie }} »^{2}$ de cet industriel comprend plusieurs éléments repris au véritable Adolphe Sax afin de faire croire à une filiation directe : le sous-titre «I8I4 Dinant * Brussels * Paris I894» 33 et les initiales A.s. entrelacées avec la marque «Belgium» dans le « $\mathrm{S}$ », à l'image du sigle A.s. «Autorisé » utilisé par les fabricants du XIxe siècle sous contrat de licence avec Adolphe Sax. ${ }^{34}$ Aux yeux de la loi, cet industriel n'a commis aucun abus, puisqu'il a le droit d'utiliser un pseudonyme comme marque, pour peu que celle-ci ne soit pas déjà déposée. Le droit et la morale ne font hélas pas toujours bon ménage, c'est là une vérité bien connue ...

Étiquettes avec dates Les dates sont généralement indiquées à la main sur les étiquettes destinées aux instruments à cordes, selon la tradition italienne. Le plus souvent, l'année est à ajouter de manière manuscrite, mais il arrive que les deux ou trois premiers chiffres soient pré-imprimés, et que le luthier la complète à la main (I8..., I89...). Ainsi, le luthier Émile Pouzol, installé à Avignon, dépose la marque « CLAUdE ROSIA / Maître Luthier / Année I9... » (fiche 788). Curieusement, ce même luthier enregistre également le même jour, à savoir le II avril I9I2, treize autres étiquettes de marques nominatives, certainement avec des noms fictifs pour appâter le client potentiel, mais chacune d'elles porte une année spécifique, alors que l'enregistrement se fait en I9I2. Donnons deux exemples: «A. STOREllo / Anno i896» (fiche 796) et « FRANCESCO MONTALDi / Anno I899» (fiche 799). Est-ce une manière d'assumer l'emploi de patronymes fictifs ?

Marques banales ou surchargées Les caractéristiques des marques examinées ci-dessus illustrent l'imagination des luthiers et facteurs d'instruments qui vont jusqu'à inventer et appliquer certaines pratiques subliminales de la publicité. À l'inverse, d'autres facteurs manquent totalement d'originalité : François Millereau présente une portée musicale avec la note sol sous l'intitulé «marque de fabrique » (fiche 20); Alphonse Blondel signe ses pianos de ses initiales, entourées d'une couronne de laurier, avec les mentions «Breveté S.G.D.G » et « Déposé » (fiche 54). De même, les pianos d'Oscar Schmidt sont marqués d'une lyre entourée de branches de laurier avec « Déposé » et « S.G.D.G. » (fiche IOI).

Une marque trop chargée peut aussi rater sa cible, car l'abondance de signes brouille toute signification. Joseph Baudassé dépose une marque destinée à recouvrir les boîtes octogonales pour cordes harmoniques. Chacune des faces porte un message, d'où une

Voir le site «Adolphe Sax et Cie», www.adolphesax.be/fr/, consulté le 27 septembre 2012. 2018).

34 Voir Malou Haine : Les licences de fabrication accordées par Adolphe Sax à ses concurrents. 26 juin I854 - I3 octobre I865, in : Revue belge de Musicologie 34/35 (I980/8I), p. I98-203. 
abondance de contenus: la mention «Chanterelles Solo», une caricature de violoncelliste, la mention «Baudassé - Cazottes / Montpellier», le dessin de l'Esplanade du Peyrou à Montpellier, seize médailles d'expositions, la mention « Diplôme d'Honneur », le monogramme BC avec une couronne de laurier, l'année I863, une petite lyre, la mention «Déposée » et aussi la photographie de la manufacture Baudassé-Cazottes (fiche 4I).

Conclusions Cette base de données de quelque I200 marques a généré trois index différents. L'index des patronymes et des noms communs recense les noms des déposants et de leurs mandataires, ainsi que les prénoms, patronymes et noms communs figurant dans les marques elles-mêmes. Le deuxième index recense les villes (autres que Paris), à la fois celles citées nommément dans les marques elles-mêmes et celles signalées comme siège d'exploitation lors des dépôts des marques auprès des tribunaux de commerce. Le troisième index détaille les éléments figuratifs contenus dans les marques (dessins, photographies, emblèmes, blasons, ornements géométriques, etc.), qui sont classées selon des critères analytiques. J'ai renoncé à établir un index des catégories de facteurs d'instruments, car, dans la plupart des cas, les spécificités mentionnées lors des dépôts sont beaucoup trop générales, de type «fabricant d'instruments de musique ». Ces indications sont toutefois reproduites telles quelles dans chacune des fiches de la base de données.

Que nous renseigne cet inventaire de près de I200 marques et ses trois index ? Il permet tout d'abord d'identifier des marques jusqu'à présent ignorées ou énigmatiques, notamment celles constituées seulement d'initiales ou d'éléments figuratifs. Les index des noms dévoilent les patronymes utilisés comme pseudonymes et, dans certains cas, les abus que cette pratique entraîne. Il complète ainsi utilement les dictionnaires et ouvrages de référence. Donnons deux exemples : la marque « Mercadier » reste une énigme pour Langwill,35 alors que la présente étude révèle qu'elle fait partie des nombreuses marques enregistrées par les facteurs Charles et Jacques Ullmann (fiche 282). L'excellent dictionnaire d'Anick Devriès et de François Lesure ne mentionne pas toutes les marques ou logos utilisés par les éditeurs recensés; ainsi, l'éditeur parisien Alphonse Michel $3^{6}$ dépose en mars I887 deux marques : « La Solidarité musicale » (fiche 95) et «A. Michel \& Rosen $\mathrm{C}^{\mathrm{ie}}$ » surmontée d'une lyre (fiche 96), indiquant par là les fondateurs de la maison. Inventors, London I993, p. 260.

36 Anick Devriès/François Lesure: Dictionnaire des éditeurs de musique français, vol.2: de I820 à I9I4, Genève I988, p.32I (notice sur Alphonse Michel). 
La base de données fournit des informations fiables relatives aux raisons sociales et aux adresses exactes à l'époque de leur dépôt, puisqu'il s'agit de documents officiels. Les noms des directeurs des maisons sont ainsi parfois également mis à jour.

La base fournit la liste des diverses marques déposées par un même facteur (les ouvrages de référence se contentent généralement d'illustrer une seule marque), dont on peut suivre aussi le passage dans des maisons successives. Ainsi, les fusions, rachats et successions apparaissent plus clairement : par exemple, la marque « Léon Bernadel » est déposée par Couesnon \& Cie, mandatés par M. Féry (fiche 94I).

Les noms des dépositaires français de marques étrangères (ou considérées comme telles) sont découverts : les pianos « BECKNER / à Paris » (fiche 2I) ou « KLEYER / PARIS » (fiche 223) ne sont ni allemands ni parisiens, mais bordelais (Louis-Jean-Alfred Ravayre, dit Raver, pour le premier ; André Delmouly, pour le second). Le piano «Vilbac / Paris » est une marque marseillaise prise par Henri Graffan (fiche 265); le guide-mains du Canadien Bohrer est exploité par Oscar Comettant (fiche 43); le métronome Maëlzel est distribué par Paquet et fils (fiche 84).

Nombreux sont les dictionnaires de luthiers, facteurs de pianos, d'instruments à vent ou d'autres domaines de la facture instrumentale qui illustrent des marques de fabrique. ${ }^{37}$ À une époque où la recherche se faisait individuellement, sans outils informatiques et sans internet, ces auteurs ont accompli un travail remarquable. Mais avouons que ces ouvrages sont quelque peu dépassés par les outils informatiques modernes : plusieurs sites internet spécialisés dans l'un ou l'autre domaine de la facture instrumentale fournissent à présent des reproductions de marques, logos et étiquettes, sites sans cesse alimentés par de nouveaux documents. Certains d'entre eux sont particulièrement riches en informations et illustrations, avec des reproductions de catalogues de vente, de la correspondance, des extraits d'archives, de presse ou des catalogues et rapports d'expositions du XIX $x^{\mathrm{e}}$ siècle. ${ }^{3}$ Les marques présentées reposent sur l'examen des instruments

37 Pour les dictionnaires d'instruments de musique, voir la nouvelle version du New Grove Dictionary of Musical Instruments, éd. Laurence Libin, London 20I4, qui a été publié aussi bien en version papier qu'en une version en ligne.

38 Parmi les nombreux sites consacrés aux luthiers et facteurs d'instruments de musique apparus ces dernières années sur la toile, un grand nombre d'entre eux sont dus à des amateurs passionnés, d'autres à des professionnels (luthiers ou musicologues). Certains se contentent d'exploiter systématiquement les ouvrages de référence publiés (sans pour autant les citer), d'autres rassemblent des informations diverses : marques et logos, catalogues de vente, archives privées ou publiques, etc. Tous permettent d'avoir un accès direct et rapide à ces informations. Nous n'en citerons que quelques-uns relatifs aux facteurs et luthiers de France, tous consultés de juillet à octobre 20I2: «Mimo : Musical Instrument Museums Online», plateforme officielle de onze musées d'instruments de musique désireux de mettre en ligne l'ensemble de leurs collections, www.mimo-db.eu/MIMO/Infodoc/; «Archéophone » recense brevets et marques de l'industrie phonographique et publie des catalogues 
parvenus jusqu'à nous, et les facteurs recensés ne sont souvent illustrés que par une seule marque, alors que mon analyse souligne leur pluralité. La difficulté majeure de ces dictionnaires ou de ces sites reste l'identification des marques composées uniquement d'initiales ou d'éléments figuratifs.

De mon côté, si la base de données et ses index recensent l'ensemble des marques déposées officiellement entre I860 et I9I9, ils ignorent totalement les marques non déposées, puisqu'il n'existe aucune obligation légale de dépôt, je le rappelle. De plus, il reste certainement plusieurs marques à découvrir dans les autres départements, même si les Archives de Paris conservent un certain nombre de duplicata de marques déposées dans d'autres villes. Malheureusement, les marques recensées ici ne font aucune distinction entre les véritables facteurs d'instruments et les marchands, sauf dans les cas où ces derniers se présentent comme tels. Ma recherche étant limitée à l'année I9I9, il serait souhaitable que d'autres chercheurs prennent la relève et étendent ce recensement jusqu'à nos jours.

Pour une meilleure connaissance des facteurs et de leurs marques, il sera toujours nécessaire de consulter divers documents : les extraits de la presse de l'époque (presse générale et presse spécialisée), les brevets d'invention, ${ }^{39}$ les catalogues de vente des firmes concernées, les archives privées et publiques, les catalogues et rapports d'exposition, ${ }^{40}$ les faillites, ${ }^{4 \mathrm{I}}$ et surtout l'examen même des instruments parvenus jusqu'à nous apportera d'utiles renseignements complémentaires. L'évolution des mentions figurant dans les marques des instruments construits par un même facteur au cours de son existence, et plus particulièrement leurs numéros de série, permettent de les dater avec plus de précision. ${ }^{2}$

en ligne, de Henri Chamoux, www.archeophone.org/; «Facteurs de pianos en France», de Lieve Verbeeck, www.lieveverbeeck.eu/pianos_francais.htm ; « Luthiers-Mirecourt » des luthiers Jean-Philippe Cognier et Rolland Terrier, www.luthiers-mirecourt.com/index.htm - Notons aussi trois blogs intéressants : «Facteurs et Marchands de Musique de l'Est de la France », http://facteursetmarchands demusique.blogspot.be ; «Luthier vents», http://luthiervents.blogspot.be ; «Archives musique, facteurs, marchands, luthiers », http://rp-archivesmusiquefacteurs.blogspot.be.

39 Lors de l'élaboration de ma thèse de doctorat au début des années I980, j'avais établi un listing de 4000 brevets et certificats d'addition relatifs à la musique, enregistrés de I798 à I900. J'ai renoncé à le publier, car ces brevets se trouvent aujourd'hui en ligne sur le site de l'INPI.

40 Voir mes travaux sur les expositions nationales et universelles: Malou Haine : Les facteurs d'instruments de musique français aux expositions nationales et universelles du xIxe siècle, publication en ligne (avril 2008), www.iremus.cnrs.fr/fr/publications/les-facteurs-dinstruments-de-musique-francais-aux-expositions -nationales-et.

41 Voir la liste des 250 faillites des facteurs d'instruments de musique dans l'ancien département de la Seine dans Malou Haine : Les faillites des facteurs d'instruments de musique. Le cas Adolphe Sax en I852, in : Musique, Images, Instruments I3 (2012), P. I47-I63.

42 Voir l'importance des numéros de série mentionnés dans les marques par l'étude d'un cas, celui des 
En complément au présent travail d'inventaire des marques recensées d'après les Archives de Paris, il serait certainement utile de consulter les documents officiels publiés par l'Institut de la Propriété Industrielle suite au dépôt des marques au greffe des tribunaux. Ma base de données pourrait alors éventuellement être complétée.

Présentation de la base de données Cette base de données comprend près de I200 marques de fabrique, enregistrées de I858 à I9I9, déposées au greffe du Tribunal de Commerce de Paris et conservées aux Archives de Paris. Ces marques concernent non seulement des fabricants et marchands habitant à Paris, mais aussi un certain nombre d'artisans ou d'industriels installés dans d'autres villes françaises ou même à l'étranger.

Les recherches peuvent s'effectuer sur un nom, un mot, une ville, un élément figuratif ou un numéro de fiche. Trois sortes d'index renvoient aux fiches individuelles : index des noms propres (patronymes) ou noms communs, index des villes, index des éléments figuratifs.

Les marques recensées concernent le domaine de la musique au sens large: instruments de musique, accessoires (cordes, anches, etc.), méthodes d'enseignement, cylindres et phonographes, instruments mécaniques. Le tableau des marques de fabrique se présente sous la forme d'un tableau réduit (numéro d'ordre, année de dépôt, nom, reproduction de la marque). ${ }^{43}$ Chaque fiche individuelle comprend huit champs, reprenant les informations figurant sur chacun des dépôts officiels au Tribunal de Commerce de Paris, à l'exception du premier champ relatif aux numéros d'ordre que je lui ai attribué :

I) Numéro d'ordre de la fiche ;

2) Date du dépôt ;

3) Nom de la firme ou, à défaut, du déposant ;

4) Spécialité ;

5) Adresse + éventuellement le nom du mandataire ou du directeur de la firme ;

6) Greffe du Tribunal de Commerce concerné et numéro du document;

instruments fabriqués par les divers membres de la famille Sax (Charles, Antoine-Joseph dit Adolphe, Alphonse, Édouard) dans Malou Haine/Ignace De Keyser: Catalogue des instruments Sax au Musée Instrumental de Bruxelles suivi de la liste de 400 instruments Sax conservés dans des collections publiques et privées, Bruxelles I980. Depuis cette publication, notre liste relative aux instruments d'Adolphe Sax et de son fils Édouard s'est considérablement et régulièrement étoffée, grâce aux travaux d'Eugenia Mitroulia et d'Arnold Myers, de l'University of Edinburgh. Leurs résultats se consultent en ligne sur le site intitulé « List of Adolphe Sax Instruments », http://homepages.ed.ac.uk/am/gdsl.html.

43 Qu'il me soit permis de remercier chaleureusement mes assistantes du Centre international pour l'Étude du XIX ${ }^{\mathrm{e}}$ siècle (Bruxelles) où j'ai travaillé jusqu'en décembre 2012 : Liliane Hermans, Vinciane Dehant et Florence Bellière ont saisi les données individuelles des fiches et établi leurs trois index. 
7) Description de la marque ;

8) Illustration de la marque.

1) Numéro d'ordre de la fiche Les fiches sont classées par ordre chronologique. Elles portent un numéro d'ordre que je leur ai attribué afin de permettre des renvois au départ des index annexés. C'est le seul élément qui ne figure pas sur les fiches officielles déposées au greffe.

2) Date des dépôts Les dépôts dont la date exacte n'a pu être déchiffrée sont rassemblés en début de l'année concernée. Le renouvellement d'un enregistrement est indiqué entre parenthèses : «(renouvellement du dépôt $n^{\circ}$.... du 20.0I.I906) ». Si la marque a fait l'objet d'un dépôt dans un autre pays, celui-ci est indiqué entre parenthèses : «(marque enregistrée en Espagne le o9. IO. I9II, sous le $\mathrm{N}^{\circ}$ I9294)».

3) Nom Ce nom peut être celui d'un fabricant, d'une société, d'une raison sociale, d'un marchand, d'un industriel, d'un négociant, etc. Dans tous les cas, le nom est celui qui identifie la maison en activité, soit sa « raison sociale ». Ce nom figure tel qu'indiqué sur la fiche officielle déposée au greffe.

Dans le cas où seul l'initial d'un prénom figure sur la fiche et que celui-ci est connu par ailleurs, il est indiqué entre crochets.

Example Fiche I9ı : J[érôme] Thibouville-Lamy.

4) Spécialité La «spécialité » est le domaine d'activités de la maison/personne concernée par la marque de fabrique : pianos, cordes, timbales, etc. Cette spécialité est celle mentionnée sur la fiche officielle déposée au greffe. Dans le cas où la spécialité n'est pas mentionnée, on l'indique entre crochets si on la connaît (en fonction d'une fiche antérieure ou postérieure): «[non précisé] » est utilisé dans les autres cas.

5) Adresse \& mandataire éventuel L'adresse est celle indiquée sur la fiche officielle déposée au greffe. S'il s'agit d'une adresse à Paris, on indique seulement la rue et le numéro : s'il s'agit d'une adresse dans une autre ville et/ou pays, on indique l'adresse complète (rue, numéro, ville, pays). Dans le cas où la personne responsable du dépôt de marque n'a pas déposé elle-même sa marque au greffe, mais qu'elle est passée par un mandataire, ce dernier est mentionné comme indiqué sur la fiche officielle déposée au greffe. Il en va de même pour celui du directeur d'une maison qui porte un patronyme différent de la maison qu'il dirige.

Example Fiche 75: Alfred Evrard, directeur de la Société anonyme des aciéries \& forges de Firminy, est celui qui dépose la marque concernée. Le patronyme «Evrard» figure dans ce champ-ci, tandis que « Société ... Firminy » est mentionné dans le champ réservé aux noms. 
6) Greffe du Tribunal de Commerce La ville du greffe concerné est indiquée dans tous les cas : il s'agit généralement de Paris, mais d'autres villes sont également concernées. Le département est mentionné comme indiqué sur la fiche officielle déposée au greffe, même s'il differe de l'appellation actuelle. Un numéro de dépôt propre au greffe suit le nom de la ville. Le numéro du cachet imprimé marqué par un tampon encreur numéroteur est aussi mentionné ici.

Example Fiche 87: marque Charles Ullmann, greffe de Paris, $n^{\circ}$ 23739, cachet imprimé $\mathrm{n}^{\circ}$ 63132I.

Un numéro de greffe illisible est indiqué : «[ill.] ; un numéro partiellement lisible comprend un «[?]» après le chiffre dont la lecture a posé problème.

Certaines marques, surtout venant de Mirecourt, possèdent deux numéros d'enregistrement. Ils sont donc indiqués :

Example Fiche 233 : Joseph Vautrin, Mirecourt $\mathrm{N}^{\circ}$ I30 et 42.

N. B. Un cachet de quatre ou cinq chiffres marqué au tampon encreur numéroteur est présent dans les marques déposées avant I89I. À partir de février I89I, il disparaît. Je l'ai donc inséré dans le champ «Greffes du Tribunal de Commerce » pour les fiches I à I43.

7) Description de la marque La description telle qu'elle figure sur la fiche officielle déposée au greffe comprend généralement trois parties :

I. Une brève description de la marque et des dessins.

Example Fiche 8I9: Dénomination «Opéra » dans une demi-couronne de roses.

2. La destination de la marque, mentionnée selon les propres termes du déposant (elle figure entre guillemets), ou résumée par moi-même (elle figure entre parenthèses):

Example Fiche 8I9 : «Destinée à désigner des cordes harmoniques de tous genres ainsi que tous instruments de musique en cuivre et en bois et tous accessoires [sic].»

Example Fiche 55 : (destinée à frapper en relief sur une fine plaque de métal à fixer sur les mécaniques de pianos).

3. Le troisième paragraphe, s'il existe, précise la manière d'appliquer la marque et donne, le cas échéant, des détails supplémentaires. Cette information est mentionnée entre parenthèses, car elle ne reprend pas textuellement les termes du déposant: Example (s'applique en creux ou en relief): (peut varier de couleur, de grandeurs ou de caractères): (à apposer comme étiquette ou de toute autre manière convenable): (à poinçonner, graver, imprimer ou apposer de toutes façons [sic]), etc. 
8) Illustration de la marque Le dernier champ reproduit la marque, sans le texte d'enregistrement qui l'encadre. Ces marques, photographiées lors de nos recherches dans les Archives de Paris, ont été ajoutées dans la base de données. En voici quelques exemples.

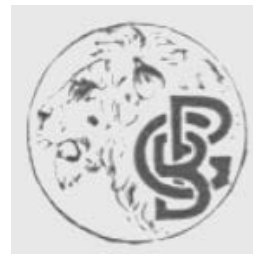

ILLUSTRATION 3 Fiche $n^{\circ} 816$ : dépôt de la marque par Pélisson, Guinot \& Blanchon, 26 avril 1912. Marque destinée à désigner tous instruments de musique

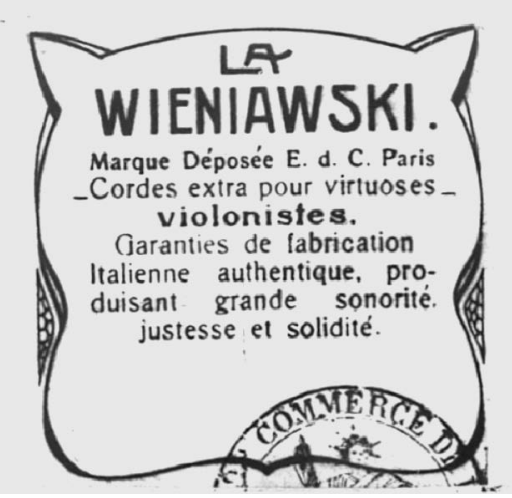

IlLUSTRATION 5 Fiche $n^{\circ} 700$ : dépôt de la marque par Édouard de Cristofaro, 19 mai 1911. Marque destinée à désigner des cordes harmoniques

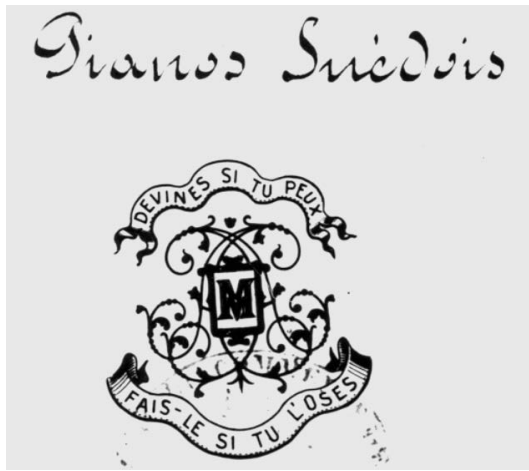

I LLUSTRATION 7 Fiche $n^{\circ} 889$ : dépôt de la marque par Édouard Joseph Mangeot, 26 mai 1885. Marque destinée à désigner des pianos

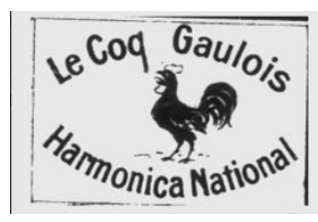

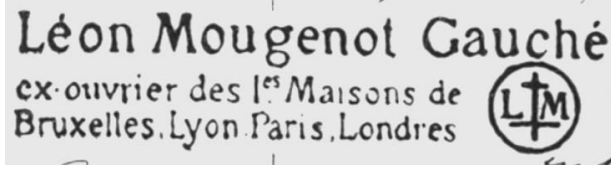

ILLUSTRATION 4 Fiche $n^{\circ} 889$ : dépôt de la marque par Léon Mougenot, 26 janvier 1913. Marque destinée à désigner tous instruments de musique

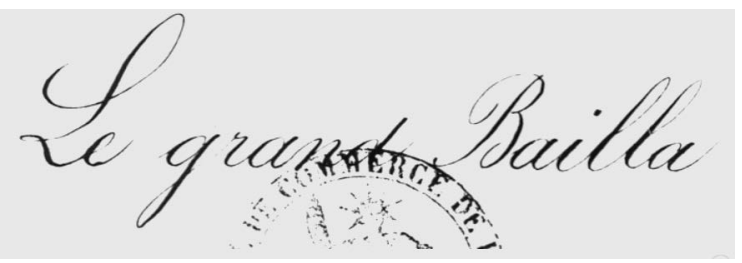

I LLUSTRATION 6 Fiche $n^{\circ} 64$ : dépôt de la marque par Émile Mennesson, 25 octobre 1881. Marque destinée au commerce de musique

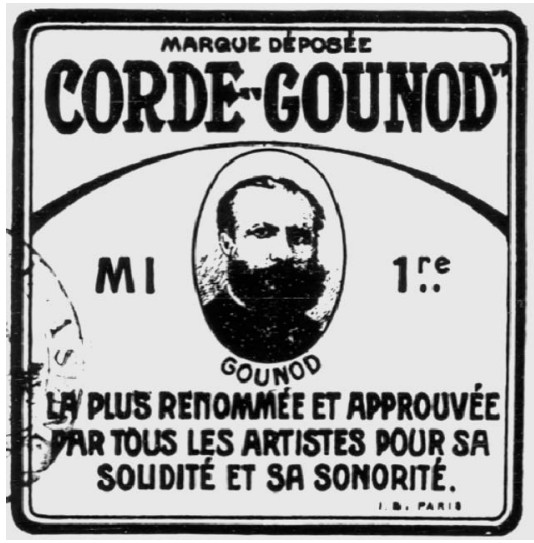

ILLUSTRATION 8 Fiche ${ }^{\circ} 1048$ : dépôt de la marque par Isaac Braudé, 13 mai 1915. Marque destinée à désigner des cordes harmoniques

IlLUSTRATION 9 Fiche ${ }^{\circ}{ }^{564}$ : dépôt de la marque par Matthieu Hohner, 25 février 1909. Marque destinée à désigner des harmonicas 


\section{Inhalt}

Vorwort 7

Adrian von Steiger "Agilité, homogénéité et beauté". The Saxhorn in the Context of the Opéra and Military Music 9

Eugenia Mitroulia/Arnold Myers The Saxhorn Families I8

Malou Haine Une nouvelle source d'archives pour identifier les marques de fabrique de facteurs d'instruments de musique (I860 à I9I9)

Stewart Carter Kastner, the Distin Family, and the Emergence of the "New" Brasswind Instruments by Adolphe Sax 68

Sabine K. Klaus Wieprecht versus Sax. German Roots of Adolphe Sax's Brasswind Designs 97

Ignace De Keyser The Construction of the Genius in

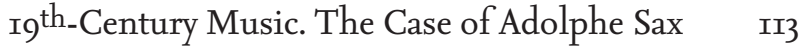

Cyrille Grenot Deux faillites d'Adolphe Sax, I873 et I877. Présentation et documents $\quad$ I46

Reimar Walthert The First Twenty Years of Saxhorn Tutors $\quad 155$

Bruno Kampmann French Makers' Improvements on Brass Instruments in the mid-I th $^{\text {th }}$ Century, Compared with Those by Adolphe Sax $\quad$ I68

Jeroen Billiet Adolphe Sax's Ultimate Masterpiece. The History, Design and Use of the Cor Sax à six pistons indépendants $\quad$ I76

Daniel Allenbach „Une réforme tellement logique« oder »à classer au rang des utopies«? Henri Chaussiers »instruments en Ut« $\quad$ I88

Gregor Widholm Das Wiener Horn - ein Instrument des I9. Jahrhunderts als erste Wahl in Orchestern des 2I. Jahrhunderts 223

Martin Skamletz »... und gar nichts, wodurch sich der eigene schöpferische Geist des Komponisten beurkundete«. Cherubini, Hummel, Konzerte, Opern, Quodlibetes und Trompeten in Wien zu Beginn des I9. Jahrhunderts.

Teil 3 und Schluss: Anton Weidinger und sein Instrument 245

Rainer Egger/Martin Mürner $\quad$ Restaurierungsergebnisse messbar machen $\quad 262$ Namen-, Werk- und Ortsregister 272

Die Autorinnen und Autoren der Beiträge $\quad 283$ 


\section{DAS SAXHORN}

Adolphe Sax' Blechblasinstrumente im Kontext ihrer

Zeit. Romantic Brass Symposium 3 - Herausgegeben von Adrian von Steiger, Daniel Allenbach und Martin Skamletz 


\section{MUSIKFORSCHUNG DER Hochschule der KÜnste Bern Herausgegeben von Martin Skamletz und Thomas Gartmann}

Band 13 
2 Dieses Buch ist in gedruckter Form im April 2020 in erster Auflage in der Edition Argus in Schliengen/Markgräflerland erschienen. Gestaltet und gesetzt wurde es im Verlag aus der Seria und der SeriaSans, die von Martin Majoor im Jahre 2000 gezeichnet wurden. Gedruckt wurde es auf Eos, einem holzfreien, säurefreien, chlorfreien und alterungsbeständigen Werkdruckpapier der Papierfabrik Salzer im niederösterreichischen Sankt Pölten. Das Vorsatzpapier Caribic cherry wurde von Igepa in Hambug geliefert. Rives Tradition, ein Recyclingpapier mit leichter Filznarbung, das für den Bezug des Umschlags verwendet wurde, stellt die Papierfabrik Arjo Wiggins in Issy-les-Moulineaux bei Paris her. Das Kapitalband mit rot-schwarzer Raupe lieferte die Firma Dr. Günther Kast aus Sonthofen im Oberallgäu, die auf technische Gewebe und Spezialfasererzeugnisse spezialisiert ist. Gedruckt und gebunden wurde das Buch von der Firma Bookstation im bayerischen Anzing. Im Internet finden Sie Informationen über das gesamte Verlagsprogramm unter www.editionargus.de, zum Institut Interpretation der Hochschule der Künste Bern unter www.hkb.bfh.ch/interpretation und www.hkb-interpretation.ch. Die Deutsche Nationalbibliothek verzeichnet diese Publikation in der Deutschen Nationalbibliografie; detaillierte bibliografische Daten sind im Internet über www.dnb.de abrufbar. (C) der zeitgleich erschienenen digitalen Version: die Autorinnen und Autoren, 2020. Dieses Werk ist lizenziert unter einer Creative Commons Namensnennung-Nicht kommerziell 4.0 International Lizenz (CC BY-NC 4.o). DOI: https://doi.org/I0.26045/kp64-6I77 ISBN 978-3-93I264-93-2 